Draft VERSion July 9, 2018

Preprint typeset using $\mathrm{LAT}_{\mathrm{E}} \mathrm{X}$ style emulateapj v. 5/2/11

\title{
WIDE FIELD MULTIBAND IMAGING OF LOW REDSHIFT QUASAR ENVIRONMENTS
}

\author{
Jennifer E. Scott ${ }^{1}$, Alireza Rafiet ${ }^{1,2}$, Jill Bechtold ${ }^{3}$, Erica Ellingson ${ }^{4}$, Christopher Thibodeau ${ }^{1,5}$, Michael \\ RICHMOND $^{1,6}$ \\ Draft version July 9, 2018
}

\begin{abstract}
We present photometry of the large scale environments of a sample of twelve broad line AGN with $0.06<z<0.37$ from deep images in the SDSS $u, g, r$, and $i$ filters taken with the 90Prime prime focus camera on the Steward Observatory Bok Telescope. We measure galaxy clustering around these AGN using two standard techniques: correlation amplitude $\left(\mathrm{B}_{g q}\right)$ and the two point correlation function. We find average correlation amplitudes for the 10 radio quiet objects in the sample equal to $(9 \pm 18,144 \pm 114,-39 \pm 56,295 \pm 260) \mathrm{Mpc}^{1.77}$ in $(u, g, r, i)$, all consistent with the expectation from galaxy clustering. Using a ratio of the galaxy-quasar cross-correlation function to the galaxy autocorrelation function, we calculate the relative bias of galaxies and AGN, $b_{g q}$. The bias in the $u$ band, $b_{g q}=3.08 \pm 0.51$ is larger compared to that calculated in the other bands, but it does not correlate with AGN luminosity, black hole mass, or AGN activity via the luminosity of the [O III] emission line. Thus ongoing nuclear accretion activity is not reflected in the large scale environments from $\sim 10 h^{-1} \mathrm{kpc}$ to $\sim 0.5 h^{-1} \mathrm{Mpc}$ and may indicate a non-merger mode of AGN activity and/or a significant delay between galaxy mergers and nuclear activity in this sample of mostly radio quiet quasars.
\end{abstract}

Subject headings: galaxies: active;large-scale structure of universe

\section{INTRODUCTION}

In local galaxies, the masses of the nuclear black holes are correlated with the stellar velocity dispersion of the host galaxy bulges, the $\mathrm{M}_{\mathrm{BH}}{ }^{-}$ $\sigma$ relation (Ferrarese \& Merritt 2000; Gebhardt et al. 2000; Tremaine et al. 2002; McLure \& Dunlop 2002; Marconi \& Hunt 2003). The host galaxies of low redshift $(z<0.4)$ quasars are consistent with these correlations, with the most powerful quasars typically inhabiting massive, early-type galaxies (McLeod \& Rieke 1995; McLeod et al.|1999; McLure et al.|1999; Hamilton et al. 2002; Dunlop et al. 2003; Floyd et al.|2004).

Theoretical modeling establishes a framework for the coevolution of galaxies and supermassive black holes such that after the initial formation of central black holes in galaxies, nuclear activity and star formation are triggered by a merger event (Barnes \& Hernquist 1992; Kauffmann \& Haehnelt 2000; Volonteri et al.|2003; Wvithe \& Loeb 2003; Granato et al. 2004; Haiman et al. 2004; Di Matteo et al. 2005; Springel et al. 2005a.,b; Croton et al. 2006; Hopkins et al. 2006a,b, 2008;

1 Department of Physics, Astronomy and Geosciences, Towson University, 8000 York Road, Towson, MD 21252-0001; [jescott,arafiee]@towson.edu

2 current address: Department of Physics and Astronomy, York University, 4700 Keele Street, Toronto, Ontario, M3J 1P3, Canada;arafiee@yorku.ca

3 Steward Observatory, University of Arizona, 933 North Cherry Ave., Tucson, AZ 85721-0065; jbechtold@as.arizona.edu

4 Center for Astrophysics and Space Astronomy, 389 UCB, University of Colorado, Boulder, CO 80309-0389; erica.ellingson@casa.colorado.edu

${ }^{5}$ current address: Department of Astronomy, University of Massachusetts, 710 North Pleasant Street, Amherst, MA 010039305;crthibodeau@astro.umass.edu

${ }^{6}$ current address: Space and Naval Warfare Systems Command Atlantic, P.O. Box 190022, North Charleston, SC 294199022
Somerville et al. 2008). These hierarchical models make specific predictions for the large scale environments of luminous quasars.

Observational studies have investigated the wide parameter space of this problem by examining: the quasarquasar and galaxy-quasar clustering in terms of correlation length and amplitude; nearest neighbor and other statistics for estimating environment density; properties of galaxies in AGN environments; and the properties of AGN host galaxies, covering also a range in redshift and intrinsic AGN properties. Many of these studies have used Sloan Digital Sky Survey (SDSS) data, thus concentrating on the $z \lesssim 0.3$ universe.

From the observational results, two points of consensus have emerged that generally agree with the models: (1) AGN activity is associated with galaxy mergers and enhanced star formation in their hosts (Sanders et al. 1988; Kauffmann et al. 2004; Guvon et al. 2006; Canalizo et al. 2007; Bennert et al. 2008; Coil et al. 2009; Veilleux et al. 2009; Teng \& Veilleux 2010), although there is increasing evidence, e.g. due to lack of correlation of galaxy structural parameters with local environment density, (Kauffmann et al. 2004) and an absence of increased rate of disturbed host morphologies in AGN hosts up to $z \sim 1.3$, Grogin et al. 2005; Gabor et al. 2009), that the onset of nuclear activity is delayed with respect to the initial galaxy interaction (Li et al. 2006, 2008; Ellison et al. 2013) and that there is a significant non-merger mode to AGN activity (Hopkins \& Hernquist 2006); (2) on megaparsec scales, bright AGN cluster like $\mathrm{L}^{*}$ galaxies (Adelberger \& Steidel 2005; Li et al. 2006; Serber et al. 2006; Coldwell \& Lambas 2006; Coil et al. 2007; Gilli et al. 2009), and over time, they occupy dark matter halos of constant mass $\left(\sim 2-3 \times 10^{12} h^{-1} \mathrm{M}_{\odot}\right)$ so they were more biased tracers of mass at higher redshift 
than they are in the current epoch (Porciani et al. 2004; Wake et al. 2004; Croom et al. 2005; Mvers et al. 2006, 2007a; Shen et al. 2007; Ross et al. 2009).

Quasar-quasar clustering shows little or no dependence on quasar luminosity (Croom et al. 2005; Porciani \& Norberg 2006; Mvers et al. 2006, 2007a; Shen et al. 2009) except at the lowest redshifts (Wake et al. 2004; Constantin \& Vogeley 2006). Galaxy clustering around AGN does correlate with nuclear activity especially for luminous, strongly accreting AGN, which tend to lie in massive hosts $\left(M^{*} \gtrsim 3 \times 10^{10} M_{\odot}\right)$ with higher than average SFR (Kauffmann et al. 2003), and are more likely to be found in lower density environments (Kauffmann et al. 2004; Constantin et al. 2008; Silverman et al. 2009; Sabater et al. 2012, 2013). No quasar luminosity dependence on galaxy-quasar clustering is seen in the DEEP2 sample with $z \sim 1$ (Coil et al. 2007), though quasars are found to cluster like blue, starforming galaxies.

AGN fueling via a merger-independent quiescent or Seyfert mode (Hopkins \& Hernquist 2006) may be most important for weaker AGN, as they show no preference for low density regions in low redshift samples (Kauffmann et al. 2004) and X-ray selected AGN at $z \sim$ 1 with lower mass hosts $\left(M^{*} \lesssim 10^{11} M_{\odot}\right)$ which are found in a broad range of environments (Silverman et al. 2009). However, there is evidence that the transition from Seyferts to LINERS proposed by Kewley et al. (2006) is affected by the density of the environment, in SDSS samples at low redshift (Constantin et al. 2008) and in the Xray selected $z \sim 1$ AGN sample of Montero-Dorta et al. (2009). These authors, and Constantin \& Vogelev (2006) also find that that the LINERS cluster more strongly than Seyfert galaxies.

Unlike galaxies, quasars do show excess clustering on small scales $\left(10-100 h^{-1} \mathrm{kpc}\right)$ up to $z \sim 3$ (Hennawi et al. 2006; Myers et al. 2007b; Shen et al. 2010). And while studies of the $\sim 1 h^{-1}$ Mpc environments around low redshift AGN show no distinct correlation between nuclear activity and the presence of galaxy neighbors on these scales (Serber et al. 2006; Coldwell \& Lambas 2006; Li et al. 2008), there is evidence that the presence of a galaxy companion on smaller scales does influence AGN activity (Alonso et al. 2007; Sabater et al. 2013). On larger scales, $z \gtrsim 1$ radio loud quasars are found in richer environments than radio quiet objects (Hall et al. 1998; Teplitz et al. 1999; Wold et al. 2003) while for low redshift this distinction is less pronounced and AGN generally are not preferentially found at the centers of rich clusters (Fisher et al. 1996; Croom \& Shanks 1999; McLure \& Dunlop 2001b; Wold et al. 2001; Coldwell et al. 2002; Barr et al. 2003; Coldwell \& Lambas 2006). For cold, or QSO, mode accretion, this can be understood as inhibition due to the stripping and harassment that occurs in these environments. However, some studies have shown that quasars do trace the large scale structure of galaxy clusters (Söchting et al. 2002, 2004; Gilli et al. 2003; Silverman et al. 2008) on scales up to $10 h^{-1} \mathrm{Mpc}$.

In this paper, we investigate the large scale environments of a sample of 12 relatively bright low redshift $(0.06<z<0.37)$ broad line AGN using deep images in the SDSS $u, g, r$, and $i$ filters. The fields presented here overlap almost entirely with the SDSS, but our photometry goes significantly deeper, in some fields and filters by up to 2.5-3 magnitudes. Our wide field multiband study on scales $\sim 1$ degree, is distinct from previous single filter studies with Hubble Space Telescope (HST) using the $\sim 3$ arcminute field of the Wide Field Planetary Camera 2 (Fisher et al. 1996; Finn et al. 2001; McLure \& Dunlop 2001b). Likewise, the multiband nature of our study extends previous wider field studies using the $\mathrm{B}_{\mathrm{J}}$ and $\mathrm{R}$ data from UK Schmidt plates (Brown et al. 2001; Coldwell et al. 2002; Söchting et al. 2002). This study is a blend of a statistical approach and a detailed study of each field. We calculate the correlation lengths and amplitudes of the galaxy-quasar clustering and compare results in different bands. We also use our data and results from the literature to discuss each quasar's environment in detail. Our primary aim is to probe galaxies as far down the luminosity function as allowed by the depth of the photometry to as wide an area around each quasar as possible in order to test if galaxy clustering around quasars is comparable to that of galaxy-galaxy clustering to these depths at these scales. Throughout this paper we assume a cosmology with $h=0.71\left(H_{0}=100 \mathrm{~h} \mathrm{~km}\right.$ $\left.\mathrm{s}^{-1} \mathrm{Mpc}^{-1}\right), \Omega_{m}=0.27$, and $\Omega_{\Lambda}=0.73$.

\section{DATA}

\subsection{Observations and Reductions}

We made our observations with the 90Prime prime focus imager (Williams et al. 2004) on the 2.3-meter Bok Telescope at Kitt Peak National Observatory in January - June, 2008. 90Prime is a wide field camera consisting of four CCDs arranged in a square mosaic located at the prime focus of the telescope with a field of view of $\sim 1$ degree. We used the SDSS $u, g, r$, and $i$ filters for the photometry, and with the exception of HS0624+690, all our fields lie inside the SDSS footprint. However, our photometry is significantly deeper than that of the SDSS survey, as discussed further in the next section. We chose the quasars in our sample to have galaxy environments accessible to deep imaging with our 2-meter class telescope and with ultraviolet spectra in the archives of the Hubble Space Telescope or Far Ultraviolet Spectroscopic Explorer for future studies of line of sight and associated absorption in these systems. The lower bound on the redshifts of the sample quasars was determined by the size of the chips in the CCD mosaic and the $\sim \mathrm{Mpc}$ scales targeted in this study. We compiled a sample of $\sim 20$ target fields for the first semester of the 2008 observing season and completed the photometry in at least two bands for 12 of those fields. Total exposure times were estimated for each field and filter in order to achieve significant detections of galaxies with $\mathrm{M}^{*}+2$ at redshift of the quasar in each filter. The final sample presented here includes one Seyfert, 9 radio quiet quasars and two radio loud quasars and so somewhat overrepresents the radio loud population, although we separate these fields out in the discussion of our results. Follow up observations to obtain data on other fields of quasars with available UV spectra and with appropriate redshifts are possible.

The quasars themselves were centered on one of the chips of the mosaic, usually chip 1, rather than in the center of the field due the $8.3^{\prime}$ interchip gaps and several traps and other quality issues with the other chips in the 
mosaic at the time of the observations. The raw data frames are multiextension FITS files consisting of eight $2048 \times 4096$ images, one for each of the two amplifiers on each chip. Amplifiers 1 and 2 lie on chip 1, 3 and 4 on chip 2, 5 and 6 on chip 3, and amps 7 and 8 are on chip 4. We give a summary of the observations in Table 1 .

We reduced the data with standard techniques for bias, zero, and flat field corrections, including fringe corrections in the $i$ band, using the standard reduction tasks in the IRAF mosaic package. 
TABLE 1

90Prime Observations

\begin{tabular}{lccccccccc}
\hline \multicolumn{1}{c}{ QSO } & Type $^{1}$ & RA & Dec & Redshift & $r$ & $u$ exp.(s) & $g$ exp.(s) & $r$ exp.(s) & $i$ exp.(s) \\
\hline MRK586 & Sy1.2 & $02: 07: 49.8$ & $+02: 42: 55$ & 0.156 & 15.42 & 2800 & 4400 & 3600 & $\ldots$ \\
HS0624+690 & QSO & $06: 30: 02.5$ & $+69: 05: 04$ & 0.370 & $14.73^{2}$ & 9200 & 6400 & 4400 & 1200 \\
PG0844+349 & Sy1.0 & $08: 47: 42.4$ & $+34: 45: 04$ & 0.064 & 14.48 & 4400 & $\ldots$ & 7600 & $\ldots$ \\
PG0923+201 & Sy1.0 & $09: 25: 54.7$ & $+19: 54: 05$ & 0.192 & 15.55 & 5600 & 5200 & 2000 & 2400 \\
PG0953+414 & Sy1.0 & $09: 56: 52.3$ & $+41: 15: 22$ & 0.234 & 14.93 & 8000 & 2400 & 3600 & 4200 \\
PG1116+215 & Sy1.0 & $11: 19: 08.7$ & $+21: 19: 18$ & 0.177 & 14.46 & 5370 & 400 & 400 & 2400 \\
PG1307+085 & Sy1.2 & $13: 09: 47.0$ & $+08: 19: 48$ & 0.155 & 15.59 & 4400 & 2000 & 3200 & 2400 \\
PG1404+226 & NLS1 & $14: 06: 21.9$ & $+22: 23: 46$ & 0.098 & 16.02 & 5200 & 4800 & 6800 & 2400 \\
PG1444+407 & Sy1.0 & $14: 46: 45.9$ & $+40: 35: 06$ & 0.267 & 15.81 & 4400 & 4000 & 3200 & 2400 \\
PG1545+210 & Sy1.2 & $15: 47: 43.5$ & $+20: 52: 17$ & 0.264 & 15.56 & 6000 & 4800 & 2800 & 2400 \\
PG1612+261 & Sy1.5 & $16: 14: 13.2$ & $+26: 04: 16$ & 0.131 & 15.87 & 4000 & 4800 & 3800 & 2400 \\
Q2141+175 & Sy1.0 & $21: 43: 35.5$ & $+17: 43: 49$ & 0.211 & 16.04 & $\cdots$ & 4500 & 3000 & 1500 \\
& & & & & & & & & \\
\hline 1 Véron-Cetty \& Véron (2010)
\end{tabular}

1 Véron-Cetty \& Véron 2010

2 Measured from 90Prime data 


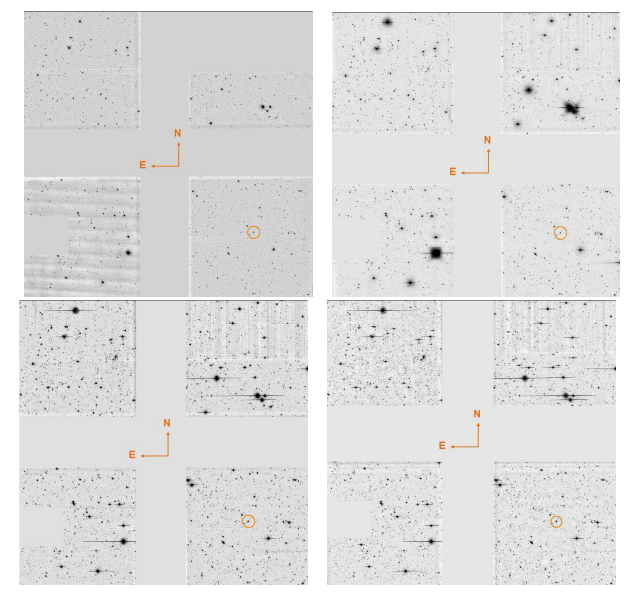

FIG. 1. - Example 90Prime data frame. The PG0923+201 field in $u, g$ (upper left, right) and $r, i$ (lower left,right).

\subsection{Photometric Calibration and Catalog Construction}

\subsubsection{Calibration and Masking}

We flux calibrated each image to the SDSS images of the same fields by selecting stars with magnitudes fainter than $\sim 15$ to avoid saturation, and brighter than $\sim 20$ where the asinh magnitude used by SDSS diverges from the traditional magnitude (Lupton et al. 1999). We used this sample to estimate the zero point magnitudes and the first order extinction coefficients for each 90Prime field and then applied these to the data. We combined the flux-calibrated images of each field to create a full exposure-time co-added image for each filter using SWarp (Bertin et al. 2002). We cross-checked star and galaxy magnitudes once again with SDSS galaxies with $r<21$ to verify the final quality of the calibration in the co-added images. Calibrated $u, g, r$, and $i$ frames for PG0923+201 are shown in Fig. 1.

HS0624+6907 does not overlap with the SDSS footprint, so to calibrate the frames of this field, we use the frames taken in each filter on photometric nights as references for the frames taken on non-photometric nights. The zero point magnitude and first order extinction coefficient is calculated from the SDSS fields taken on the same photometric nights, and so this photometry is also tied to the SDSS system as the rest of the sample. We calculated photometric, Kron, and Petrosian magnitudes using the SExtractor package (Bertin \& Arnouts 1996) on the full exposure-time flux-calibrated co-added images of each filter. We found that optimal source extraction was achieved by considering each amplifier section of each image on a case-by-base basis for amps 1-8 and the extraction parameters used thus vary slightly over the full mosaic. We give a brief summary of those parameter choices here. Amps 1, 2, and 3, are typically low noise and free of bad columns. So we used $1.5 \sigma$ for detection and analyze thresholds, DETECT_THRESH and ANALYZE_THRESH. Since the fields are crowded, we use a balanced combination of background and deblending parameters, BACK_SIZE, DEBLEND_NTHRESH, and DEBLEND_MINCONT, to detect as many individual objects and as close as possible to stars or foreground galaxies but to avoid over-resolving. Amp 4 has noise patterns which are enhanced by resampling. To over-

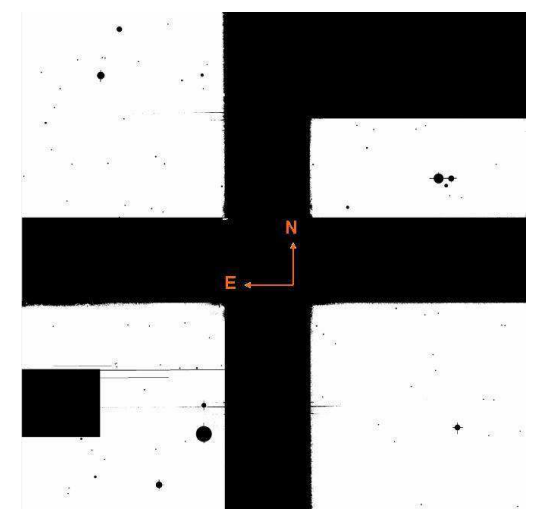

Fig. 2.- Example of a frame mask, for the PG0923+201 in u filter, shown in Fig. 1 including inter-chip gaps, trap in amps 5 and 6 , bad pixel/columns, and bright stars.

come this problem we used a moderately higher detection threshold, $\sim 3$, and larger area to estimate the background with deblending parameters set to identify the strips as artificial bright sources. In some extreme cases we mask the noise pattern manually. Amps 5 and 6 have a higher noise level than amps 1 and 2 but typically free of fixed patterns, so we use the same technique as amp 1 but with a higher detection threshold, 1.7 . Amps 7 and 8 behave generally like amps 5 and 6 . We dealt with a few cases of noise patterns using the same technique as amp 4 , with detection a threshold of $\sim 2.3$.

To remove bad columns and bright stars and their associated diffraction patterns, we created image masks which consist of: (1) a manually generated mask for the interchip gaps; (2) a mask for a consistent trap present in chip 3, overlapping amps 5 and 6; (3) a bright star mask created manually by identifying the bright stars in a given frame from the SExtractor output and growing the masked regions using the measured FWHM; (4) a bad pixel mask created using the IRAF task objmasks to mask pixels with pixel values $15 \sigma$ below the background; and finally, (5) a bleed trail mask created by using the segmentation map created by SExtractor for a detection threshold $\sim 80-100$ and very low debelending parameter and large background estimates, thus including all the bleed patterns. We created masks for each image of each field in every filter individually. The downside of this technique is that step (5) can include some bright foreground galaxies into the mask, so we recover these from careful inspection of the images in all four filters. As an example, our mask frame for PG0923+201 in $u$ filter (Fig. 1) is shown in Fig. 2 Due to the large dithering of individual frames before co-adding, some regions have lower co-added exposure time than the full stacked image. To decrease the biases on galaxy counting when using a certain detection limit on magnitude, we cut the corners and more from inner-gaps to include only the regions with uniform co-added exposure time in each final image. We also mask objects with centers within 8 pixels of a masked region or an edge from the final catalogs. Finally we filter our catalog sources based on the internal SExtractor flags. Here, we present the catalog of objects detected at $5 \sigma$ or greater in $r$ and at $3 \sigma$ or greater in $u, g$ and $i$ in Table 2 as a machine readable table. The magnitudes listed are the SExtractor MAG_BEST values, where the Kron_fact and min_radius parameters gov- 
erning MAG_AUTO were set to 2.5 and 1.5, respectively. Magnitudes of 99 in this table denote non-detections in the $u, g$, or $i$ filter, and values of -99 label objects not observed in one of those filters, primarily due to masking. In the former case, the error bar gives the $1 \sigma$ detection limit for later use with photometric redshift codes. For the analyses discussed in this paper, we use only galaxies detected at $5 \sigma$ significance or greater. 
TABLE 2

90Prime Catalog of Galaxies in Quasar Fields

\begin{tabular}{|c|c|c|c|c|c|c|c|c|c|c|c|c|c|c|c|c|c|c|}
\hline QSO field & RA & Dec & $u^{1}$ & $\sigma_{u}$ & $g$ & $\sigma_{g}$ & $r$ & $\sigma_{r}$ & $i$ & $\sigma_{i}$ & $\mathrm{~A}^{2}$ & $\sigma_{\mathrm{A}}$ & $\mathrm{B}^{2}$ & $\sigma_{\mathrm{B}}$ & radius $^{3}$ & $\theta^{3}$ & $\mathrm{FWHM}^{3}$ & class $^{3}$ \\
\hline HS0624p690 & 98.0779 & 68.8485 & 23.10 & 0.14 & 99.00 & 25.52 & 21.20 & 0.08 & 20.65 & 0.11 & 1.443 & 0.109 & 0.840 & 0.064 & 2.56 & -12.91 & 5.05 & 0.02 \\
\hline HS0624p690 & 98.1246 & 68.8445 & 99.00 & 27.74 & 99.00 & 25.52 & 22.67 & 0.18 & 99.00 & 25.54 & 0.677 & 0.143 & 0.519 & 0.110 & 7.79 & -75.60 & 2.93 & 0.00 \\
\hline HS0624p690 & 97.2567 & 68.8409 & 99.00 & 27.74 & 99.00 & 25.52 & 21.93 & 0.11 & 21.28 & 0.16 & 0.852 & 0.121 & 0.531 & 0.075 & 5.67 & -85.21 & 2.72 & 0.61 \\
\hline HS0624p690 & 98.0474 & 68.8475 & 99.00 & 27.74 & 99.00 & 25.52 & 20.80 & 0.06 & 19.90 & 0.08 & 1.213 & 0.080 & 0.863 & 0.057 & 3.97 & 21.11 & 2.96 & 0.96 \\
\hline HS0624p690 & 97.5498 & 68.8449 & 99.00 & 27.74 & 99.00 & 25.52 & 21.03 & 0.07 & 99.00 & 25.54 & 0.964 & 0.069 & 0.920 & 0.065 & 5.46 & -35.21 & 2.37 & 0.26 \\
\hline HS0624p690 & 97.6265 & 68.8442 & 99.00 & 27.74 & 99.00 & 25.52 & 22.11 & 0.13 & 22.12 & 0.23 & 0.799 & 0.116 & 0.603 & 0.088 & 5.97 & 61.78 & 2.45 & 0.57 \\
\hline HS0624p690 & 97.4941 & 68.8537 & 99.00 & 27.74 & 99.00 & 25.52 & 21.78 & 0.11 & 21.71 & 0.19 & 0.981 & 0.118 & 0.682 & 0.082 & 5.02 & -82.06 & 3.82 & 0.12 \\
\hline HS0624p690 & 98.1444 & 68.8458 & 99.00 & 27.74 & 99.00 & 25.52 & 21.85 & 0.11 & 20.80 & 0.12 & 0.799 & 0.107 & 0.562 & 0.075 & 5.90 & 73.74 & 2.28 & 0.73 \\
\hline HS0624p690 & 97.5849 & 68.8437 & 99.00 & 27.74 & 99.00 & 25.52 & 22.75 & 0.17 & 22.25 & 0.24 & 0.535 & 0.129 & 0.432 & 0.104 & 6.39 & 59.89 & 2.28 & 0.50 \\
\hline HS0624p690 & 97.7454 & 68.8461 & 99.00 & 27.74 & 99.00 & 25.52 & 21.79 & 0.11 & 20.74 & 0.12 & 0.858 & 0.109 & 0.740 & 0.094 & 5.77 & 29.80 & 2.87 & 0.10 \\
\hline HS0624p690 & 97.8299 & 68.8482 & 99.00 & 27.74 & 99.00 & 25.52 & 21.17 & 0.08 & 20.56 & 0.11 & 1.254 & 0.098 & 0.846 & 0.066 & 3.67 & 46.78 & 3.74 & 0.21 \\
\hline HS0624p690 & 97.5091 & 68.8468 & 99.00 & 27.74 & 21.62 & 0.10 & 21.40 & 0.09 & 21.00 & 0.14 & 0.942 & 0.086 & 0.733 & 0.067 & 4.76 & -29.61 & 2.48 & 0.86 \\
\hline HS0624p690 & 97.5560 & 68.8461 & 99.00 & 27.74 & 99.00 & 25.52 & 22.41 & 0.15 & 99.00 & 25.54 & 0.642 & 0.111 & 0.559 & 0.097 & 6.31 & 18.58 & 2.16 & 0.64 \\
\hline HS0624p690 & 98.0133 & 68.8473 & 99.00 & 27.74 & 99.00 & 25.52 & 22.77 & 0.18 & 21.12 & 0.14 & 0.753 & 0.166 & 0.462 & 0.102 & 5.87 & -88.26 & 3.53 & 0.23 \\
\hline HS0624p690 & 97.3695 & 68.8452 & 99.00 & 27.74 & 99.00 & 25.52 & 22.90 & 0.18 & 22.11 & 0.23 & 0.704 & 0.156 & 0.423 & 0.094 & 5.34 & 75.93 & 3.2 & 0.52 \\
\hline HS0624p690 & 97.3791 & 68.8490 & 99.00 & 27.74 & 99.00 & 25.52 & 20.78 & 0.06 & 99.00 & 25.54 & 1.245 & 0.079 & 0.874 & 0.055 & 5.03 & 28.43 & 2.92 & 0.20 \\
\hline HS0624p690 & 97.9586 & 68.8496 & 99.00 & 27.74 & 99.00 & 25.52 & 22.51 & 0.16 & 21.22 & 0.15 & 0.571 & 0.125 & 0.490 & 0.107 & 6.94 & 75.39 & & 0.49 \\
\hline HS0624p690 & 97.5345 & 68.8568 & 19.66 & 0.03 & 18.32 & 0.02 & 17.46 & 0.01 & 17.10 & 0.02 & 2.327 & 0.029 & 2.117 & 0.027 & 3.37 & -74.43 & 3.91 & 0.03 \\
\hline HS0624p690 & 97.5649 & 68.8494 & 99.00 & 27.74 & 99.00 & 25.52 & 22.12 & 0.13 & 21.59 & 0.18 & 0.542 & 0.155 & 0.378 & & 8.39 & -13.78 & 2.36 & 0.36 \\
\hline HS0624p690 & 97.0202 & 68.8484 & 99.00 & 27.74 & 99.00 & 25.52 & 20.98 & 0.07 & 20.17 & 0.09 & 1.610 & 0.121 & 0.900 & 0.068 & 4.66 & 54.87 & 4.81 & 0.39 \\
\hline HS0624p690 & 97.8307 & 68.8524 & 99.00 & 27.74 & 99.00 & 25.52 & 21.14 & 0.08 & 20.04 & 0.09 & 0.858 & 0.065 & 0.760 & & 4.07 & 79.73 & 2.00 & 0.96 \\
\hline HS0624p690 & 97.1526 & 68.8478 & 99.00 & 27.74 & 99.00 & 25.52 & 22.41 & 0.15 & 99.00 & 25.54 & 0.676 & 0.147 & 0.501 & 0.109 & 7.64 & -77.44 & 3.48 & 0.47 \\
\hline HS0624p690 & 97.9007 & 68.8552 & 99.00 & 27.74 & 99.00 & 25.52 & 20.11 & 0.05 & 19.11 & 0.06 & 1.381 & 0.062 & 0.896 & 0.040 & 3.47 & 9.03 & 3.23 & 0.94 \\
\hline HS0624p690 & 97.7168 & 68.8546 & 99.00 & 27.74 & 99.00 & 25.52 & 20.83 & 0.07 & 20.13 & 0.09 & 1.116 & 0.072 & 0.897 & 0.058 & 4.24 & -75.39 & 2.69 & 0.53 \\
\hline HS0624p690 & 97.8514 & 68.8552 & 99.00 & 27.74 & 99.00 & 25.52 & 21.59 & 0.10 & 21.05 & 0.14 & 0.912 & 0.125 & 0.780 & 0.107 & 6.80 & 0.23 & 4.54 & 0.00 \\
\hline HS0624p690 & 97.9116 & 68.8545 & 99.00 & 27.74 & 99.00 & 25.52 & 22.63 & 0.16 & 21.52 & 0.17 & 0.705 & 0.127 & 0.522 & 0.094 & 4.74 & 74.68 & 3.43 & 0.64 \\
\hline HS0624p690 & 98.0572 & & 22.87 & 0.13 & 99.00 & 25.52 & 21.29 & 0.09 & 20.81 & 0.12 & 1.592 & 0.142 & 0.783 & & 4.98 & 87.82 & 5.97 & 0.00 \\
\hline HS0624p690 & 97.6051 & 68.8607 & 99.00 & 27.74 & 99.00 & 25.52 & 22.02 & 0.12 & 22.28 & 0.24 & 0.863 & 0.114 & 0.664 & 0.088 & 5.13 & 85.51 & 2.90 & 0.12 \\
\hline
\end{tabular}

${ }^{1}$ MAG_BEST from SExtractor

2 semimajor and semiminor axes in arcsec

3 SExtractor parameters KRON_RADIUS, THETA_J2000 (in degrees), FWHM, and CLASS_STAR as discussed in the text and in Bertin et al. (2002) 
TABLE 3

Flux Calibration Errors

\begin{tabular}{lcccc}
\hline \hline filter & Systematic err. & $95 \%$ conf. & Total & $95 \%$ conf. \\
\hline$u$ & -0.039 & 0.011 & 0.305 & 0.009 \\
$g$ & -0.083 & 0.006 & 0.152 & 0.005 \\
$r$ & -0.028 & 0.008 & 0.122 & 0.007 \\
$i$ & -0.022 & 0.013 & 0.066 & 0.006 \\
& & & & \\
\hline
\end{tabular}

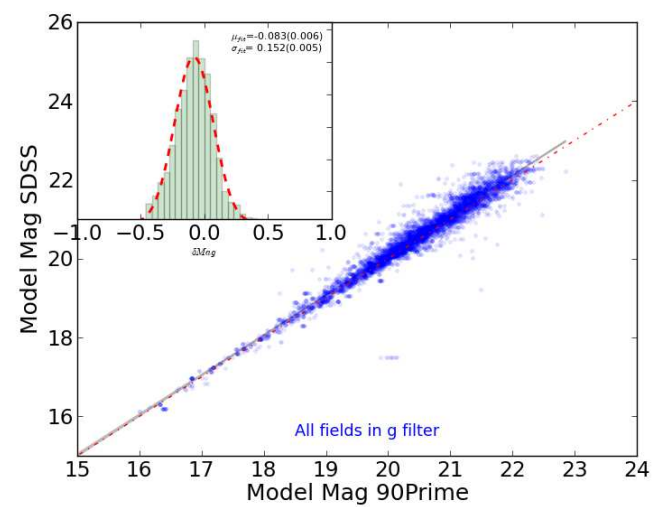

FIG. 3.- Example of error estimates from photometric calibration process in $\mathrm{g}$ filter from all fields.

We estimate systematic error and scatter due photometric calibration for all four filters using a final comparison with the SDSS photometry. For this comparison, we use all objects detected in our catalogs at $\geq 5 \sigma$ and with a SExtractor classification parameter (CLASS_STAR, described in the next section) likely to be a galaxy, $<0.3$. We also require the relative error in the SDSS magnitude to be smaller than $1 \%$. The values of the systematic shifts are small, typically less than 0.04 . They are listed in Table 3 and plotted for the $g$ band in Fig. 3 Also in Table 3 we list the $95 \%$ confidence on the systematic offset, along with the total photometric statistical uncertainties in each band and their $95 \%$ confidence interval. The total statistical uncertainties are obtained from adding the systematic offsets from SDSS in quadrature with the standard deviation of the distribution of SExtractor magnitude errors for each filter. The mean and standard deviation of those distributions are listed separately for each field in Table 4 .

\subsubsection{Star-Galaxy Separation and Filtering}

SExtractor calculates a CLASS_STAR flag based on core geometry of the light distribution of the detected objects, where values near 1 indicate a centrally concentrated star and those near 0 indicate a galaxy. This parameter is sensitive to the value of the input parameter SEEING_FWHM. We measure this input parameter from objects clearly classified as stars (CLASS_STAR > 0.9) in initial photometric catalogs, which were created from a first pass with SExtractor on the masked images. To improve the accuracy of the CLASS_STAR star-galaxy separation output parameter, we performed this measurement iteratively until we reached convergence between the input value of the SEEING_FWHM and the measured FWHM of the stellar catalog sources. We achieved convergence of $<0.1^{\prime \prime}$ in 1-2 iterations. In order to deter-
TABLE 4

Individual Flux Calibration Errors

\begin{tabular}{|c|c|c|c|c|c|}
\hline field & filter & Systematic err. & $95 \%$ conf. & Total & $95 \%$ conf \\
\hline MRK586 & $u$ & -0.061 & 0.016 & 0.328 & 0.012 \\
\hline MRK586 & $g$ & -0.026 & 0.012 & 0.253 & 0.010 \\
\hline MRK586 & $r$ & 0.007 & 0.016 & 0.139 & 0.014 \\
\hline PG0844+349 & $u$ & 0.009 & 0.013 & 0.293 & 0.010 \\
\hline PG0844+349 & $r$ & -0.031 & 0.008 & 0.131 & 0.007 \\
\hline PG0923+201 & $u$ & 0.003 & 0.013 & 0.356 & 0.011 \\
\hline PG0923+201 & $g$ & -0.062 & 0.012 & 0.169 & 0.011 \\
\hline PG0923+201 & $r$ & 0.022 & 0.012 & 0.144 & 0.010 \\
\hline PG0923+201 & $i$ & 0.119 & 0.020 & 0.199 & 0.016 \\
\hline PG0953+414 & $u$ & 0.005 & 0.011 & 0.286 & 0.009 \\
\hline PG0953+414 & $g$ & -0.030 & 0.008 & 0.134 & 0.006 \\
\hline PG0953+414 & $r$ & -0.008 & 0.010 & 0.114 & 0.009 \\
\hline PG0953+414 & $i$ & 0.062 & 0.013 & 0.131 & 0.010 \\
\hline PG1116+215 & $u$ & -0.019 & 0.013 & 0.317 & 0.010 \\
\hline PG1116+215 & $g$ & -0.029 & 0.025 & 0.118 & 0.015 \\
\hline PG1116+215 & $r$ & -0.019 & 0.041 & 0.070 & 0.019 \\
\hline PG1116+215 & $i$ & 0.095 & 0.018 & 0.171 & 0.014 \\
\hline $\mathrm{PG} 1307+085$ & $u$ & 0.034 & 0.010 & 0.292 & 0.008 \\
\hline PG1307+085 & $g$ & -0.046 & 0.010 & 0.148 & 0.008 \\
\hline $\mathrm{PG} 1307+085$ & $r$ & -0.014 & 0.006 & 0.071 & 0.005 \\
\hline PG1307+085 & $i$ & 0.067 & 0.018 & 0.145 & 0.012 \\
\hline PG1404+226 & $u$ & -0.117 & 0.016 & 0.376 & 0.013 \\
\hline $\mathrm{PG} 1404+226$ & $g$ & -0.076 & 0.010 & 0.172 & 0.008 \\
\hline PG1404+226 & $r$ & -0.032 & 0.008 & 0.134 & 0.007 \\
\hline PG1404+226 & $i$ & 0.064 & 0.022 & 0.171 & 0.018 \\
\hline PG1444+407 & $u$ & -0.083 & 0.014 & 0.284 & 0.012 \\
\hline PG1444+407 & $g$ & -0.089 & 0.008 & 0.148 & 0.007 \\
\hline PG1444+407 & $r$ & -0.036 & 0.018 & 0.111 & 0.016 \\
\hline PG1444+407 & $i$ & 0.006 & 0.013 & 0.145 & 0.011 \\
\hline PG1545+210 & $u$ & -0.005 & 0.007 & 0.331 & 0.006 \\
\hline PG1545+210 & $g$ & -0.091 & 0.014 & 0.165 & 0.013 \\
\hline PG1545+210 & $r$ & -0.017 & 0.011 & 0.093 & 0.008 \\
\hline PG1545+210 & $i$ & 0.036 & 0.017 & 0.161 & 0.014 \\
\hline PG1612+261 & $u$ & -0.088 & 0.014 & 0.305 & 0.011 \\
\hline PG1612+261 & $g$ & -0.110 & 0.010 & 0.128 & 0.008 \\
\hline PG1612+261 & $r$ & -0.045 & 0.011 & 0.108 & 0.008 \\
\hline PG1612+261 & $i$ & 0.033 & 0.012 & 0.185 & 0.010 \\
\hline $\mathrm{Q} 2141+175$ & $g$ & -0.093 & 0.010 & 0.155 & 0.007 \\
\hline $\mathrm{Q} 2141+175$ & $r$ & -0.058 & 0.012 & 0.100 & 0.011 \\
\hline $\mathrm{Q} 2141+175$ & $i$ & 0.010 & 0.021 & 0.185 & 0.014 \\
\hline
\end{tabular}

mine how to use this CLASS_STAR parameter to best distinguish stars from galaxies in our final catalogs, we performed Monte-Carlo simulations of our data set using synthetic input stars that we then extract from the data frames using the same SExtractor parameters as we used on the data. Using the IRAF task mkobjects, we generated synthetic stars with magnitudes between 15 and 27 and with PSFs that matched those of stellar objects in the post-iteration catalogs. We randomly distributed these synthetic stars on chips 1 and 4 (amps 1,2,7, and 8 ) of each image, to bracket the best and worst quality CCDs in the mosaic. After extracting these simulated stars, we see the typical behavior of the CLASS_STAR parameter, i. e. that it is a reliable star-galaxy separator at bright magnitudes but at a particular threshold magnitude, CLASS_STAR is spread evenly between 1.0 and some saturation value, 0.35 for our 90Prime frames. Thus, at magnitudes dimmer than the threshold, we cannot rely on this parameter alone to separate galaxies from stars. This threshold magnitude varies across our data frames, but for each frame, we fit a function to the lower envelope of CLASS_STAR versus magnitude, $m$ :

$$
\text { CLASS_STAR }=a /(1.0+\exp ((m-b) / c))+d
$$




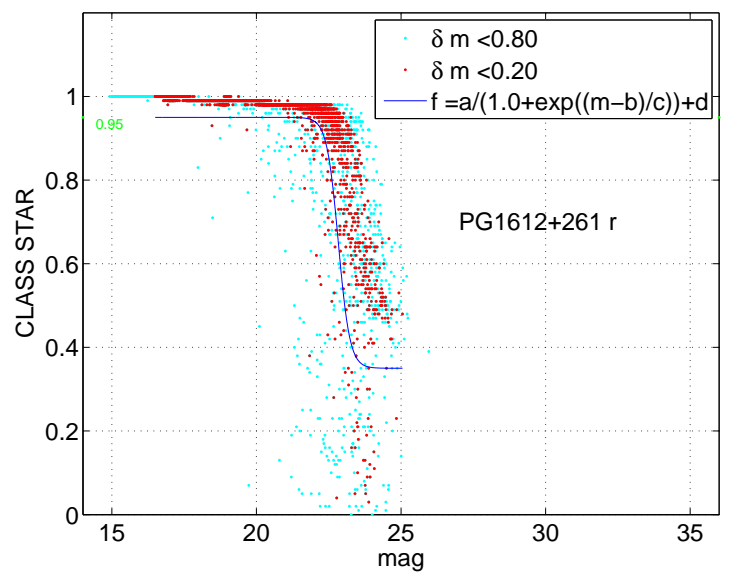

FIG. 4.- CLASS_STAR versus $\mathrm{r}$ for PG1612+261 with the fit to Equ. 1 Cyan(red) points are points for which the difference between the input and output stellar magnitudes in the MonteCarlo simulations was less than $0.8(0.2)$. The fit is designed to find the best inner envelope for the red data reaching asymptotic value of 0.95 at lowest magnitude.

where $m$ is the magnitude in the relevant filter, and $a$, $b, c$, and $d$ are fitted parameters. We use the fits to this expression in our final star-galaxy separation algorithm. Figure 4 shows an example of this fitting.

Equ. 1 is used to define a cut in the CLASS_STAR parameter as function of source magnitude. We find that using this function as a strict cut results in many faint sources lost from our catalogs, so we relax this criterion and rely on a color based criterion as well. To construct the color criterion, we use the unambiguous stellar sources in the matched catalogs, those with CLASS_STAR $>0.95$ in all filters, along with the sources in the Gunn-Stryker Atlas (Gunn \& Stryker 1983) to define the stellar locus in color-color space. We then exclude from our catalogs any source that lies within a specified distance from that locus in both $g-r$ versus $u-g$ and $r-i$ versus $g-r$. This distance is allowed to vary in order to give the best match in number counts to our adopted luminosity functions as described below, but it is typically 0.1-0.3. In order to use the color information for sources with only one available color, we use a technique similar to that described by Coil et al. (2004). The binned distributions of the stars in our catalogs in each color, normalized to have a maximum value of unity, are used to assign a probability of being a star, $p_{s}$. Objects having a $p_{s}$ above a threshold value are excluded from the final galaxy catalogs. This threshold is also allowed to vary slightly, typically between 0.7 and 0.8 , to get good agreement in the number counts in each filter. The resulting number counts from these final catalogs, shown below, give us confidence in this star-galaxy separation technique. In Figure 5 we show the positions of the $5 \sigma$ galaxies in $u, g, r$, and $i$ in the portion of the field of PG1444+407 covered by chip 1, along with the position of the quasar itself.

\subsubsection{Limiting Magnitudes}

We characterize the depth of our data frames in two ways: (1) by finding the magnitude at which the lower envelope of the distribution of magnitude errors for stars in the catalogs equals 0.217 , or $5 \sigma$ and (2) by using the magnitude distribution of all catalog objects detected at

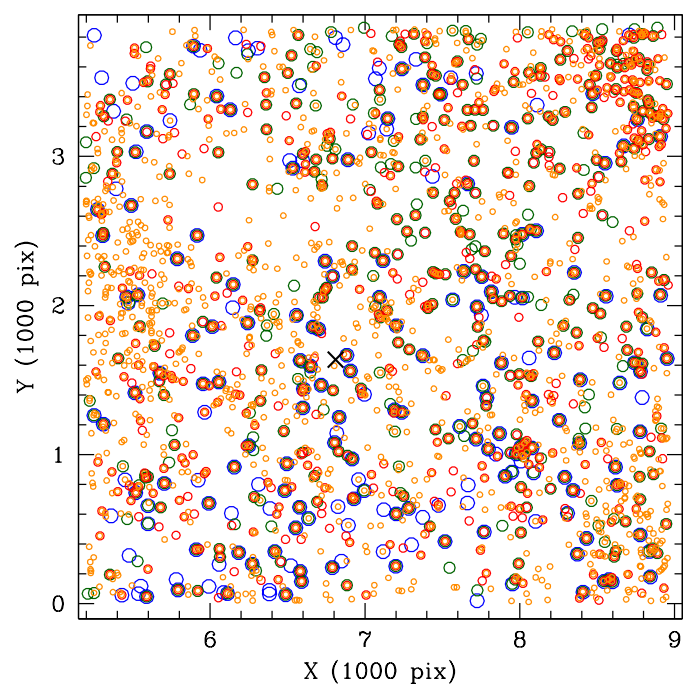

FIG. 5.- Blue, green, red, and orange circles show the positions of galaxies detected in $u, g, r$, and $i$ at $>5 \sigma$ significance with magnitude brighter than the limiting magnitude listed in column 3 of Table 5 for chip 1 of the PG1444+407 field. Black cross marks the position of PG1444+407 itself.

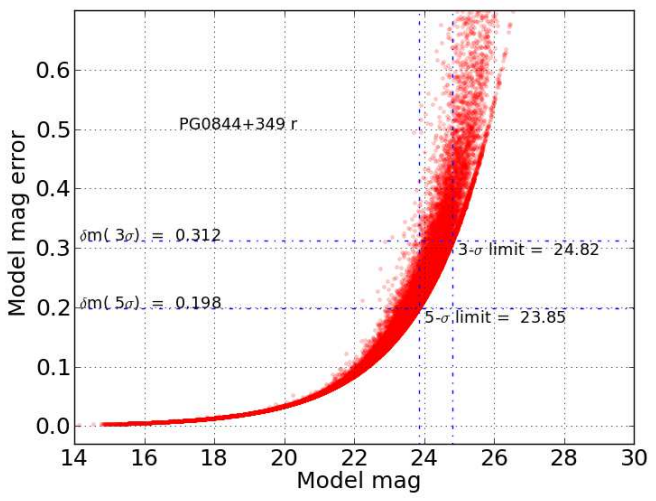

FIG. 6.- Example of method (1) for calculating limiting magnitudes at various significance levels.

$>4.5 \sigma$. We show examples of each in Figures 6 and 7 and tabulate these values for each co-added frame in Table 5. Because we used slightly modified SExtractor parameters for the different amps of the full mosaic, we calculated the limiting magnitudes separately for each amp using method (1). We find that the values for all other amps agree with that of amp 1 to within $<0.03$ magnitudes on average, so we report only values for that amplifier.

We treat method (1) as our estimator of the limiting magnitude at a particular level of significance. Because we use stars only for method (1), it is affected by our ability to recover faint extended galaxies from the images. Method (2) therefore best characterizes the depth of the galaxy catalogs, since those objects dominate the number counts at the faintest magnitudes, assuming our stargalaxy separation technique is robust. We discuss this further below, in Section 3 .

Results for each field/filter with each method are listed in Table 5. For reference, the SDSS limiting magnitudes 
TABLE 5

LiMiting MAGNitudes

\begin{tabular}{ccccc}
\hline \hline QSO & filter & $\begin{array}{c}5 \sigma \mathrm{m}_{\text {lim }} \\
\text { method }(1)\end{array}$ & $\begin{array}{c}3 \sigma \mathrm{m}_{\text {lim }} \\
\text { method }(1)\end{array}$ & $\begin{array}{c}\text { m(complete) } \\
\text { method }(2)\end{array}$ \\
\hline HS0624+6907 & $u$ & 23.8 & 23.9 & 22.9 \\
HS0624+6907 & $g$ & 21.9 & 21.9 & 20.7 \\
HS0624+6907 & $r$ & 23.1 & 23.4 & 22.1 \\
HS0624+6907 & $i$ & 22.0 & 23.1 & 21.7 \\
MRK586 & $u$ & 22.9 & 24.0 & 22.5 \\
MRK586 & $g$ & 23.6 & 24.5 & 22.9 \\
MRK586 & $r$ & 23.3 & 24.4 & 23.1 \\
PG0844+349 & $u$ & 23.9 & 24.5 & 23.3 \\
PG0844+349 & $r$ & 24.0 & 25.1 & 23.3 \\
PG0923+201 & $u$ & 23.7 & 24.8 & 23.5 \\
PG0923+201 & $g$ & 23.6 & 24.7 & 23.3 \\
PG0923+201 & $r$ & 22.7 & 23.8 & 22.5 \\
PG0923+201 & $i$ & 22.8 & 24.0 & 22.3 \\
PG0953+414 & $u$ & 24.1 & 25.2 & 23.9 \\
PG0953+414 & $g$ & 22.9 & 24.1 & 22.9 \\
PG0953+414 & $r$ & 23.3 & 24.4 & 23.1 \\
PG0953+414 & $i$ & 23.6 & 24.7 & 22.7 \\
PG1116+215 & $u$ & 23.7 & 24.8 & 23.5 \\
PG1116+215 & $g$ & 22.0 & 23.0 & 21.7 \\
PG1116+215 & $r$ & 22.2 & 23.3 & 22.1 \\
PG1116+215 & $i$ & 22.8 & 23.9 & 22.5 \\
PG1307+085 & $u$ & 23.5 & 24.6 & 23.3 \\
PG1307+085 & $g$ & 22.7 & 23.9 & 22.7 \\
PG1307+085 & $r$ & 23.1 & 24.2 & 23.1 \\
PG1307+085 & $i$ & 22.9 & 24.0 & 22.7 \\
PG1404+226 & $u$ & 23.6 & 24.6 & 23.3 \\
PG1404+226 & $g$ & 23.6 & 24.8 & 23.5 \\
PG1404+226 & $r$ & 24.0 & 25.1 & 23.5 \\
PG1404+226 & $i$ & 22.8 & 23.9 & 22.5 \\
PG1444+407 & $u$ & 23.4 & 24.6 & 23.3 \\
PG1444+407 & $g$ & 23.4 & 24.6 & 23.3 \\
PG1444+407 & $r$ & 23.2 & 24.4 & 23.1 \\
PG1444+407 & $i$ & 22.8 & 24.0 & 22.7 \\
PG1545+210 & $u$ & 23.8 & 24.9 & 23.5 \\
PG1545+210 & $g$ & 23.5 & 24.7 & 23.3 \\
PG1545+210 & $r$ & 23.1 & 24.2 & 22.9 \\
PG1545+210 & $i$ & 22.8 & 23.9 & 22.3 \\
PG1612+261 & $u$ & 23.3 & 24.4 & 23.1 \\
PG1612+261 & $g$ & 23.9 & 25.0 & 23.7 \\
PG1612+261 & $r$ & 23.6 & 24.7 & 23.5 \\
PG1612+261 & $i$ & 23.1 & 24.3 & 22.9 \\
Q2141+175 & $g$ & 23.8 & 24.9 & 23.7 \\
Q2141+175 & $r$ & 23.4 & 24.5 & 23.3 \\
Q2141+175 & $i$ & 22.6 & 23.8 & 22.3 \\
\hline & & & &
\end{tabular}

in $(u, g, r, i)$ are $(22.0,22.2,22.2,21.3)$, corresponding to $95 \%$ completeness limits. These values may be compared with column 4 , where we list the $3 \sigma$ limiting magnitudes found via method (1). The stacked $g$ band image of the HS0624+6907 field was created from a combination of data taken on photometric and non-photometric nights, calibrated as described above. The resuling image in this particular field and filter had a resulting level of noise that necessitated a high detection threshold within SExtractor to avoid numerous spurious detections around bright sources. Thus, the 3 - and $5 \sigma$ limiting magnitudes are reported here to be the same, as there are no sources extracted at less than $4 \sigma$. This also results in a shallow field compared to the other photometry reported here. Note that the limiting magnitudes in the 90Prime data are significantly fainter than SDSS in most other fields and filters.

\section{NUMBER COUNTS}

In Figure 8 we show the galaxy number counts in all four bins from our 90Prime fields, over a total of 7.35, $7.45,8.95$, and $7.03 \mathrm{deg}^{2}$ in $u, g, r$, and $i$, respectively.

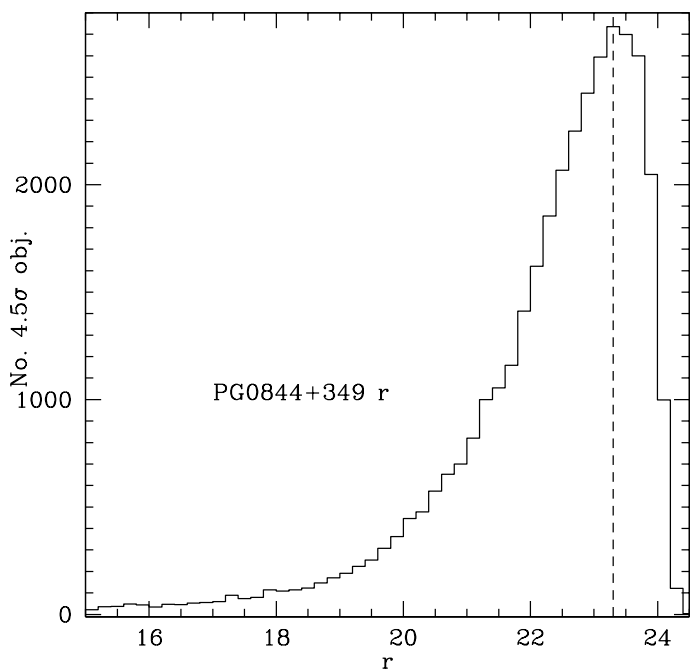

FIG. 7.- Example of method (3) for calculating completeness using the magnitude distribution of all catalog objects detected at $>4.5 \sigma$

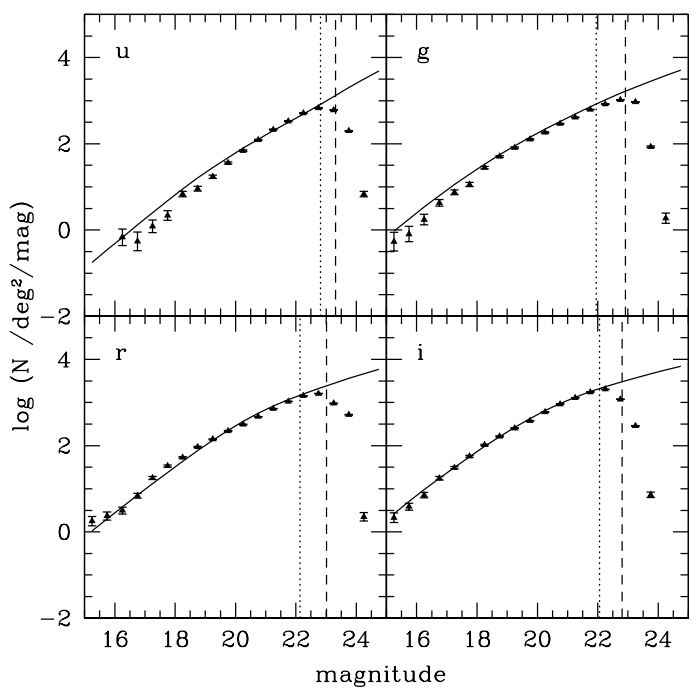

FIG. 8. - Number of galaxies in 0.5 magnitude bins per square degree in our 90Prime fields in $u, g, r$, and $i$. Solid lines are the number counts predicted by the luminosity function in each band. The vertical dotted and dashed lines show the limiting magnitudes for Samples 1 and 2 respectively, listed in Table 6 and discussed in Section 4

The solid lines in Figure 8 show the predicted number of galaxies for our adopted luminosity functions in each band. Luminosity function parameters adopted are shown in Fig. 9. The curves shown here are polynomial fits to the parameter values derived as a function of redshift from several surveys: in $u$, we adopt the parameters found by Prescott et al. (2009) from the DEEP2 and SDSS $u$-band Galaxy Survey; and for the $r$ band, we adopt the parameters found by Cool et al. (2012) from the AGES survey. For the $g$ band, we adopt the $B$ band values from the DEEP2 survey (Faber et al. 2007), using the $g$ band luminosity function parameter measurements of Loveday et al. (2012) to constrain our fits at $z=0$. We make this choice because: (a) the wavelength 


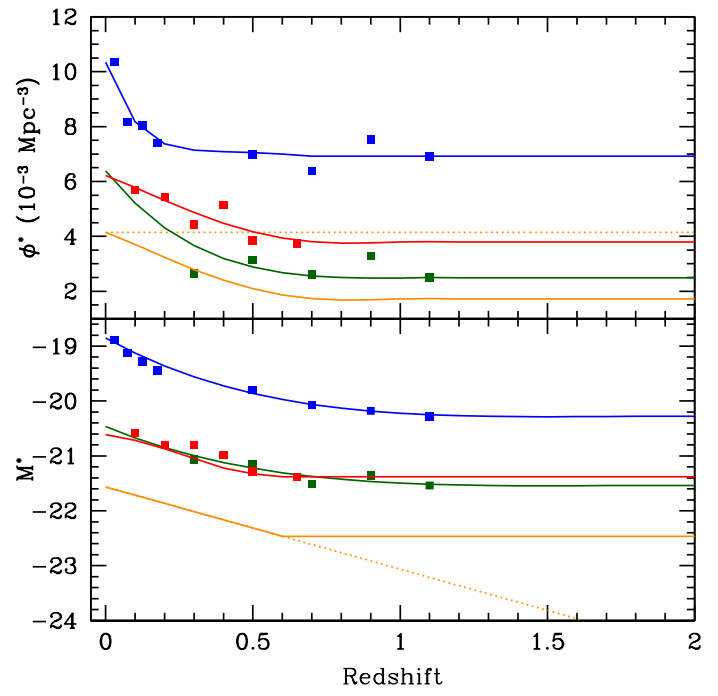

FIG. 9.- Polynomial fits (solid curves) to luminosity function parameter values $\phi^{*}$ and $M^{*}$ (squares) from Prescott et al. (2009) $(u$ band, blue), (Faber et al. 2007) ( $B$ band, green), Cool et al. (2012) ( $r$ band, red), and For $i$ band (orange) the Lovedav et al. (2012) parametrized model for $M^{*}$ is set at the $z=0.6$ value for larger redshifts, and for $\phi^{*}$ it follows the redshift evolution of the $r$ band from its $z=0$ value. Dashed curves show Lovedav et al. (2012) parametrized models for the redshift evolution of each parameter in $i$ extrapolated to $z>0.5$.

coverage of $B$ overlaps that of the SDSS $g$ filter; (b) the agreement between these B band luminosity function parameters and the directly measured parameters in $g$ from Lovedav et al. (2012) is good at $z<0.5$; and (c) while no AGN with $z>0.5$ are considered in this work, the Faber et al. (2007) study provides a more robust and convenient way to parametrize the luminosity function to redshifts beyond $z=0.5$ than an extrapolation of the Loveday et al. (2012) measurements, enabling us to estimate the numbers of background galaxies reliably. For the $i$ band, we adopt a modification of the evolution derived by Loveday et al. (2012). The $M^{*}$ follows the parametric solution found by these authors, but we fix its value at $z>0.6$ to the $z=0.6$ value. For $\phi^{*}$, we find good agreement with our galaxy number counts by using the Loveday et al. (2012) value at $z=0$ and adopting the redshift evolution for the $r$ band, noting that the Loveday et al. (2012) parametrized solution found no redshift evolution in this band. The $M^{*}$ and $\phi^{*}$ evolution in $i$ found by Loveday et al. (2012) and extrapolated to $z>0.5$ is shown in Fig. 9. For $\alpha$, the values from these various studies are $(-1.0,-1.3,-1.05$, -1.12) for $(u, B, r, i)$. We extrapolate the highest redshift values from each study to all higher redshifts for our calculations, although at the depths of our survey, we are generally not sensitive to galaxies with $z \gtrsim 1.5$.

\section{CORRELATION FUNCTIONS}

To examine the galaxy autocorrelation function and the galaxy-quasar cross-correlation signal in the 90Prime fields, we use a chip-by-chip approach to the calculations. Due to the large interchip gaps in the 90Prime mosaic, the masked region in chip 3, and the often masked amplifier 4 on chip 2 (see Fig. 2), the correlation functions described below contain significant structure when cal-
TABLE 6

SAMPLE Limiting MAGNitudes

\begin{tabular}{lll}
\hline \hline Filter & $\mathrm{m}_{\lim }$ & $\mathrm{M}_{\text {lim }}-\mathrm{M}^{* 1}$ \\
\hline \multicolumn{3}{c}{ Sample 1} \\
\hline$u$ & 22.82 & -1.1 \\
$g$ & 21.95 & -0.8 \\
$r$ & 22.14 & 0.3 \\
$i$ & 22.06 & 1.6 \\
\hline \multicolumn{3}{c}{ Sample 2 } \\
\hline$u$ & 23.31 & -0.6 \\
$g$ & 22.92 & 0.2 \\
$r$ & 23.01 & 1.1 \\
$i$ & 22.81 & 2.3 \\
\multicolumn{3}{c}{ For $z=0.5$} \\
\hline
\end{tabular}

culated at scales that span these regions, even with a large simulated set of random points. To verify this, we performed a series of simulations with 5000 random data points created with regions identical to those in the real data. With the random data, we expect zero correlation but find that there is significant structure in the correlation function when the masks are applied, and so we calculate the correlation functions in the real data on scales that minimize the effect of the masks introducing structure into the correlation functions that is unrelated to the galaxy distributions.

To perform the correlation function calculations to a uniform depth, we confine galaxy magnitudes to the limit defined by the shallowest field in the sample. These depths are listed in Table 6 for the two cases we consider: Sample 1 is the sample of all quasar fields with the HS0624+6901 $g$ band excluded due to its shallow depth $(g=21.79)$ and Sample 2 is a sample of deeper fields, constructed by leaving out other shallow fields in each filter to achieve the depth listed in Table 6. The Sample 1 and 2 limiting magnitudes are also shown as vertical dotted and dashed lines in Figure 8 . In our calculations, we will consider other AGN in the primary sample fields with redshifts up to $z=0.5$. Thus, in Table 6 we also list the absolute magnitudes relative to $\mathrm{M}^{*}$ reached for $z=0.5$ galaxies in Samples 1 and 2 in order to demonstrate the coverage of the galaxy luminosity function at the redshifts of the AGN in the overall sample.

\subsection{Galaxy-Galaxy Clustering}

We calculate the angular correlation function of the galaxy-galaxy sample,

$$
\omega(\theta)_{\mathrm{true}}=A_{\mathrm{gg}} \theta^{(1-\gamma)}
$$

using the estimator of Landy \& Szalav (1993):

$$
\omega(\theta)_{\mathrm{obs}}=\frac{N_{\mathrm{gg}}(\theta)-2 N_{\mathrm{gr}}(\theta)-N_{\mathrm{rr}}(\theta)}{N_{\mathrm{rr}}(\theta)}
$$

where $N_{\text {gg }}$ and $N_{\text {rr }}$ are the pairwise distances between all galaxies in the sample and all random positions in the survey area at a given angular separation, $\theta$, normalized by the total numbers of galaxy-galaxy and randomrandom pairwise distances. The $N_{\mathrm{gr}}$ term is the normalized number of pairwise distances between all galaxies and random points in each $\theta$ bin. 
The random points are generated by Monte Carlo simulations which place galaxies at random positions within the areas covered by the 90Prime images. These catalogs of random positions are masked in the same way as the galaxy catalogs. We generated 10 realizations of each field in each filter, with $\sim 40,000$ points in each realization. The Poisson error in each bin is given by

$$
\Delta \omega(\theta)=\frac{1+\omega(\theta)}{\sqrt{N_{\mathrm{gg}}(\theta)}} .
$$

This estimator of the angular correlation function must be corrected for the integral constraint, which requires the function to reach zero at the survey edges, so that

$$
\omega(\theta)_{\text {true }}=\omega(\theta)_{\mathrm{obs}}+C
$$

where

$$
C=\frac{1}{\Omega^{2}} \iint \omega(\theta) d \Omega d \Omega=\frac{\sum N_{\mathrm{rr}}(\theta) A_{\mathrm{gg}} \theta^{(1-\gamma)}}{\sum N_{\mathrm{rr}}(\theta)}
$$

(Groth \& Peebles 1977; Infante 1994).

In each filter, we solve for $\mathrm{A}_{\mathrm{gg}}$ and $\gamma$ independently using a least squares technique and derive the uncertainties in the parameters by finding the values of each that yield $\chi_{\min }^{2}+1$.

\subsection{Galaxy-Quasar Clustering}

For galaxy-quasar clustering, the autocorrelation function does not apply, so we follow Croom \& Shanks (1999) and Brown et al. (2001)

$$
\omega(\theta)_{\text {true }}=\omega(\theta)_{\mathrm{obs}}+C=\frac{N_{\mathrm{gq}}(\theta)}{N_{\mathrm{qr}}(\theta)}-1+C
$$

where $N_{\mathrm{gq}}$ and $N_{\mathrm{qr}}$ are the numbers of galaxy-quasar and quasar-random pairs at each value of $\theta$, normalized by the numbers of galaxies and random points respectively and $C$ is defined as in Equ. 6

Here we solve for $\mathrm{A}_{g q}$ for a fixed value of $\gamma$ and again derive the uncertainties in the parameters by finding the values of each that yield $\chi_{\min }^{2}+1$.

\subsubsection{Correlation Length}

The galaxy-galaxy and the galaxy-quasar angular correlation functions can be used to derive the correlation length, $r_{0}$, of the spatial correlation function

$$
\xi=\left(\frac{r}{r_{0}}\right)^{\gamma}(1+z)^{-(3+\epsilon)}
$$

for galaxy-galaxy and galaxy-quasar clustering using Limber's equation (Limber 1953), which relates $r_{0}$ to the amplitude of the angular correlation function given in Equation 2

$$
A=k r_{0}^{\gamma} \frac{\int_{0}^{\infty} F(z) D_{\theta}^{(1-\gamma)}(z) N_{x}(z) N_{g}(z) g(z) d z}{\int_{0}^{\infty} N_{x}(z) N_{g}(z) d z}
$$

where

$$
k=\sqrt{(\pi)} \frac{\Gamma\left(\frac{\gamma-1}{2}\right)}{\Gamma\left(\frac{\gamma}{2}\right)}
$$

and $F(z)$ describes the redshift evolution of the correlation function in Equ. 8 with $\epsilon=0$ or $\gamma-3$ for clustering

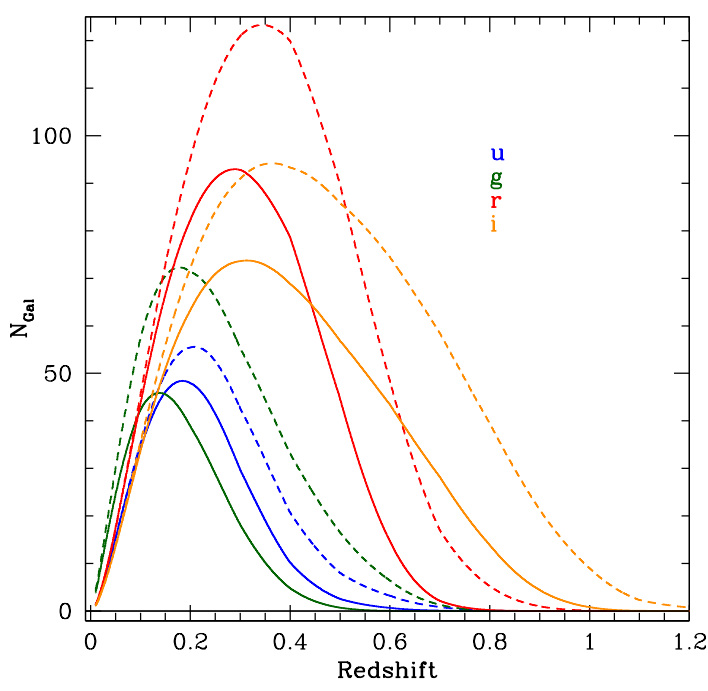

FIG. 10. - Number of galaxies in $u, g, r, i$ frames in all 90Prime fields from integration of a Schechter luminosity function with parameters as shown in Figure 9] integrated to the limiting magnitudes listed in column 3 of Table 5 Solid lines show Sample 1 and dashed lines represent Sample 2.

fixed in physical or comoving coordinates, or $\epsilon=\gamma-1$ for clustering according to linear theory. Finally,

$$
g(z)=\frac{H_{0}}{c}\left[(1+z)^{3}\left(1+\Omega_{m} z+\Omega_{\Lambda}\left((1+z)^{-2}-1\right)^{\frac{1}{2}}\right]\right.
$$

and $N_{x}$ corresponds to either $N_{g}$ or $N_{q}$ depending on whether we are considering galaxy-galaxy or galaxyquasar clustering.

The redshift distribution of the galaxies in our 90Prime Samples 1 and 2, calculated from the Schechter luminosity function parameters shown in Figure 9 and the magnitude limits determined by the values listed in Table 6 in each of the four bands is shown in Figure 10.

\subsubsection{Correlation Amplitude}

We also calculate the amplitude of the galaxyquasar correlation function, $\mathrm{B}_{g q}$ using the technique described by Ellingson et al. (1991), Finn et al. (2001), McLure \& Dunlop (2001b), and recently by Ramos Almeida et al. (2013). We first begin with the angular correlation function, which describes the clustering of galaxies about some particular object using the following relation:

$$
n(\theta) d \Omega=N_{g}[1+w(\theta)] d \Omega .
$$

Here, $n(\theta) d \Omega$ represents the number of galaxies within a solid angle $\mathrm{d} \Omega$ at an angular distance $\theta$ from the object. The term $N_{g}$ represents the average galaxy surface density, and $w(\theta)=A_{g q} \theta^{1-\gamma}$ represents the angular crosscorrelation function discussed above. To calculate $\mathrm{B}_{g q}$, we first determine the amplitude, $\mathrm{A}_{g q}$, from the integral of the angular correlation function within a fixed angular radius, $\theta$,

$$
A_{g q}=\frac{N_{t}-N_{b}}{N_{b}} \frac{(3-\gamma)}{2} \theta^{\gamma-1},
$$

where $N_{t}$ represents the quasar field galaxy counts, and $N_{b}$ represents the background galaxy counts both within 
angular distance $\theta$ of the quasar. For each quasar field, we count the galaxies within a specified radius around the quasar, down to the magnitude limit specified by the amplifiers on the chip, $m_{\text {lim }}$. To construct the control fields, we use all other regions of the mosaic of the reference quasar as well as any non-quasar regions of the images of our other quasar fields. We place grids of overlapping control fields with the same radius as our reference quasar in each of these regions and count the galaxies brighter than $m_{\text {lim }}$. The galaxy counts in these regions may be affected by other galaxy clusters and large scale structures in the quasar fields. We discuss these in detail for each quasar field in Appendix A. To mitigate this problem we take a median of all the counts from these regions and use this as our value for $N_{b}$.

The angular covariance amplitude, $\mathrm{A}_{g q}$, quantifies any excess in galaxy counts within the quasar field as compared with the background counts of the control field. To de-project the angular cross-correlation function into its spatial equivalent, we use:

$$
n(r) d V=\rho_{g}[1+\xi(r)] d V,
$$

where $n(r)$ and $\rho_{g}$ represent the total and expected average galaxy counts within volume $d V$, respectively, and the spatial cross-correlation function, $\xi(r)$, is defined as in Equ. 8, with amplitude $\mathrm{B}_{g q}$ :

$$
\xi(r)=\mathrm{B}_{g q} r^{-\gamma},
$$

where $\gamma=1.77$ (Groth \& Peebles 1977). $\quad \mathrm{B}_{g q}$ can be obtained using the following equation relating the spatial clustering amplitude to the angular clustering amplitude (Longair \& Seldner 1979):

$$
\mathrm{B}_{g q}=\frac{N_{g} A_{g q}}{\Phi\left(m_{\lim }, z\right) I_{\gamma}}\left[\frac{D}{1+z}\right]^{\gamma-3} .
$$

Here, $D$ is the angular diameter distance to the quasar, and $\phi(z)$ is the galaxy luminosity function integrated to the limiting absolute magnitude of the data at the quasar redshift. The quantity $I_{\gamma}$ is a constant of integration equivalent to $\frac{2^{\gamma}}{(\gamma-1)} \frac{\Gamma(\gamma+1 / 2)}{\Gamma(\gamma)}$.

The errors in $\mathrm{A}_{g q}$ and $\mathrm{B}_{g q}$ can be calculated using the following equations:

$$
\frac{\Delta A_{g q}}{A_{g q}}=\frac{\Delta B_{g q}}{B_{g q}}=\frac{1}{N_{t}-N_{b}}\left[\left(N_{t}-N_{b}\right)+1.3^{2} N_{b}\right]^{\frac{1}{2}} .
$$

(Yee \& López-Cruz 1999). We normalize the galaxy counts using the luminosity function calculated with the redshift-dependent parameters shown in Fig. 9.

To calculate the predicted average galaxy background counts, $N_{g}$, we integrate the luminosity function using the same redshift-dependent parameters:

$$
N_{g}=\int_{0}^{z} \int_{-\infty}^{M_{\mathrm{lim}}} \Phi(m, z) d m d z
$$

to the relevant luminosity dictated by the limiting magnitude of the data at each redshift. In calculating each value of $M_{\text {lim }}$, we apply a median K-correction for galaxies in each filter at each redshift calculated from the KCORRECT code (Blanton \& Roweis 2007) and using the galaxy templates of Kinney et al. (1996).
In our data sample, we adjust the galaxy magnitudes for extinction using galaxy extinction $\mathrm{E}(\mathrm{B}-\mathrm{V})$ values acquired from the NASA/IPAC Infrared Science Archive for each quasar, and assuming $R_{V}=3.1$ and the reddening curve of O'Donnell (1994). In our calculations, we used a radius of $0.5 h^{-1} \mathrm{Mpc}$ around the sample quasars and for the control fields derived from the other 90Prime images of comparable depth in the same filter, and we fix the parameter $\gamma$ to be 1.77. We follow Finn et al. (2001) and McLure \& Dunlop (2001b) in using only galaxies in the range $m^{*}-1-m^{*}+2$ in order to eliminate many galaxies in the foreground or background of the quasar of interest.

\section{RESULTS}

\subsection{Comparisons with other work}

\subsubsection{Correlation Amplitude}

Results of the calculations of $\mathrm{B}_{g q}$ are listed in Table 7 and plotted versus quasar redshift in Figure [11, along with values for some of our fields present in the literature. We do not include a value for PG0844+349, as its low redshift, $z=0.064$, results in an angular radius corresponding to $0.5 h^{-1} \mathrm{Mpc}$ that is larger than our contiguous chip areas. We do include this object in our discussion of the angular correlation function below. We also omit the $i$ band data of Q2141+175 from both this calculation and the correlation function calculations as the quasar was placed in amp 4, which was masked due to noise.

The limiting magnitudes of the galaxies included in these calculations are those listed in Table [5] with the exception of PG0953+414 $u$ and $i$ bands (23.7 and 23.2, respectively), PG1404+226 $i$ (22.5), and PG1612+261 $g(23.5)$, as there were no galaxies found in the control fields in these bands down to the quoted magnitude limits. This change in the magnitude limits had no effect on $N_{t}$, the quasar field galaxy counts, but simply increased $N_{b}$, the median number of background galaxies found in the control fields to a number greater than zero, resulting in a finite value of $\mathrm{B}_{g q}$. No significant differences in the $\mathrm{B}_{g q}$ values were found when imposing a uniform magnitude limit on all the fields, so we opt for using the individual values for each field. All $\mathrm{B}_{g q}$ values are quoted in units of $\mathrm{Mpc}^{\gamma}$, where $\gamma=1.77$ unless otherwise noted. The mean values of $\mathrm{B}_{g q}$ we find in $(u, g, r, i)$ are $(21 \pm 20$, $161 \pm 96,58 \pm 100$, and $445 \pm 271)$.

Given the well-known morphology-density relation (Dressler 1980) and the fact that radio loud quasars are more commonly found in massive early-type hosts (Hamilton et al. 2002; Best et al. 2005), it may be expected that galaxies cluster more strongly around radio loud quasars than around radio quiet objects. Optical and X-ray selected AGN show no tendency to reside in galaxy group environments (Coldwell \& Lambas 2003; Silverman et al. 2009) but Wold et al. (2001) find that radio loud quasars do prefer group or Abell class 0 environments. Some other studies have found this distinction between the environments of radio loud and radio quiet quasars (Ellingson et al. 1991; Best et al. 2005; Shen et al. 2009; Hickox et al. 2009; Ramos Almeida et al.|2013) while others have not (Fisher et al.|1996; McLure \& Dunlop 2001b; Finn et al. 2001; Wold et al. 2001; Coldwell et al. 2002). As we have 
only 2 radio loud quasars in our sample, we have little leverage to address this question. However, excluding the two radio loud objects in our sample, PG1545+210 and Q2141+175, we find a systematic decrease in all four filters: $<\mathrm{B}_{g q}>=(9 \pm 18,144 \pm 114,-39 \pm 56,295 \pm 260)$. Removing the two additional $u$ fields (PG1444+407 and HS0624+690) and one $g$ field (HS0624+690) that do not have limiting magnitudes that reach $\mathrm{M}^{*}+2$ at the redshifts of the AGN does not affect these means significantly, bringing them to $32 \pm 14$ and $202 \pm 113$ respectively. The error bars reflect the standard deviation in the measurements among all fields in each filter. The median redshift of our sample AGN is 0.18 and the median $\mathrm{M}_{r}$ is -23.7. These modified $\mathrm{B}_{g q}$ averages are all consistent with the galaxy-galaxy correlation amplitude, 20 $\left(h^{-1} \mathrm{Mpc}\right)^{1.77}$ (Davis \& Peebles 1983), although within the uncertainties, the average values in $g$ and $i$ are also consistent with richer environments, as discussed in more detail for individual fields below.

Finn et al. (2001) used images of quasar fields taken with the F606W, F675W, and F702W filters and the 2.5' field of view of the Wide Field Planetary Camera 2 on the Hubble Space Telescope and, scaling to our assumed value of $\mathrm{H}_{0}$, find $\left\langle\mathrm{B}_{g q}\right\rangle=104 \pm 22,-33 \pm 27$, and $133 \pm 44$ in those respective filters for radio quiet quasars at median redshifts of $0.17,0.42$ and 0.4 . McLure \& Dunlop $(2001 \mathrm{~b})$ find $\left\langle\mathrm{B}_{g q}\right\rangle=326 \pm 94$ for their sample of radio quiet objects with median redshift of 0.16 , also derived from $\mathrm{F} 606 \mathrm{~W}$ and $\mathrm{F} 675 \mathrm{~W}$ HST/WFPC2 images. Their dataset is a superset of that of Fisher et al. (1996) who found $\left\langle\mathrm{B}_{g q}\right\rangle=132 \pm 37$. These $H S T$ studies were limited to radii of $\sim 200 \mathrm{kpc}$, while ground-based studies typically extend to the same $0.5 h^{-1} \mathrm{Mpc}$ scale as ours does. The ground-based V, R, and I photometry of Wold et al. (2001) targets AGN at higher redshift than our AGN sample, $z_{\text {med }}=0.714$, and they find $\left\langle\mathrm{B}_{g q}\right\rangle=210 \pm 82$ for radio quiet quasars. All of these studies show average $\mathrm{B}_{g q}$ values that are larger than $\mathrm{B}_{g g}$ at the $2-3 \sigma$ level. Our overall results are more in line with the earlier studies of Smith et al. (1995) and Ellingson et al. (1991), both with $z_{\text {med }} \sim 0.4$ who found average $\mathrm{B}_{g q}$ values consistent with $\mathrm{B}_{g g}$ for radio quiet objects.

Considering the individual values, we find that there can be a large range of $\mathrm{B}_{g q}$ values obtained for the same field depending on the filter used. Typically, the values of $\mathrm{B}_{g q}$ in the $i$ band tend to be larger than those of the other bands and those calculated in the $u$ band are nearly all consistent with $\mathrm{B}_{g g}$. As above, for the $r$ band, we compare our individual results with those found in the literature, from Finn et al. (2001) and McLure \& Dunlop (2001b), and for PG1545+210, we compare with Yee \& Green (1984), who calculated $\mathrm{B}_{g q}$ from $r$ band images taken with the SIT-vidicon camera and the $1.52 \mathrm{~m}$ Palomar telescope. We scaled these literature $\mathrm{B}_{g q}$ values for our value of $\mathrm{H}_{0}$ but otherwise do not correct for different cosmologies used in these other works (McLure \& Dunlop (2001b): $H_{0}=50 \mathrm{~km}$ $\mathrm{s}^{-1} \mathrm{Mpc}^{-1}$; Finn et al. (2001) $H_{0}=100 \mathrm{~km} \mathrm{~s}^{-1} \mathrm{Mpc}^{-1}$, both use $\left.\Omega_{\mathrm{m}}=1, \Omega_{\Lambda}=0\right)$. In most cases our results are consistent with these previous studies. We give a more detailed discussion of these comparisons for each field in Appendix A.

Yee \& Ellingson (1993) considered $\quad \mathrm{B}_{g q}>\quad 500$ to
TABLE 7

$\mathrm{B}_{g q}$ Measurements

\begin{tabular}{|c|c|c|c|}
\hline QSO & $\mathrm{B}_{g q}\left(\mathrm{Mpc}^{\gamma}\right)^{1}$ & Filter & Ref. $^{2}$ \\
\hline \multirow[t]{5}{*}{ MRK586 } & $-3 \pm 6$ & $u$ & 1 \\
\hline & $-36 \pm 137$ & $g$ & 1 \\
\hline & $0^{3}$ & $r$ & 1 \\
\hline & $93 \pm 161$ & F606W & 2 \\
\hline & $13 \pm 82$ & F606W & 3 \\
\hline \multirow[t]{4}{*}{ HS0624+6907 } & $-66 \pm 52$ & $u$ & 1 \\
\hline & $-319 \pm 279$ & $g$ & 1 \\
\hline & $-144 \pm 127$ & $r$ & 1 \\
\hline & $-126 \pm 151$ & $i$ & 1 \\
\hline \multirow[t]{6}{*}{ PG0923+201 } & $-76 \pm 25$ & $u$ & 1 \\
\hline & $-114 \pm 133$ & $g$ & 1 \\
\hline & $-377 \pm 130$ & $r$ & 1 \\
\hline & $-529 \pm 291$ & $i$ & 1 \\
\hline & $500 \pm 269$ & F675W & 2 \\
\hline & $119 \pm 81$ & F606W & 3 \\
\hline \multirow[t]{6}{*}{ PG0953+414 } & $30 \pm 28$ & $u$ & 1 \\
\hline & $207 \pm 101$ & $g$ & 1 \\
\hline & $-36 \pm 191$ & $r$ & 1 \\
\hline & $629 \pm 519$ & $i$ & 1 \\
\hline & $730 \pm 297$ & F675W & 2 \\
\hline & $159 \pm 81$ & F606W & 3 \\
\hline \multirow[t]{6}{*}{ PG1116+215 } & $68 \pm 41$ & $u$ & 1 \\
\hline & $-41 \pm 57$ & $g$ & 1 \\
\hline & $186 \pm 134$ & $r$ & 1 \\
\hline & $863 \pm 461$ & $i$ & 1 \\
\hline & $321 \pm 235$ & F606W & 2 \\
\hline & $185 \pm 81$ & F606W & 3 \\
\hline \multirow[t]{6}{*}{ PG1307+085 } & $10 \pm 27$ & $u$ & 1 \\
\hline & $259 \pm 120$ & $g$ & 1 \\
\hline & $125 \pm 306$ & $r$ & 1 \\
\hline & $1461 \pm 778$ & $i$ & 1 \\
\hline & $112 \pm 176$ & F606W & 2 \\
\hline & $170 \pm 82$ & F606W & 3 \\
\hline \multirow[t]{4}{*}{ PG1404+226 } & $85 \pm 85$ & $u$ & 1 \\
\hline & $942 \pm 522$ & $g$ & 1 \\
\hline & $143 \pm 759$ & $r$ & 1 \\
\hline & $707 \pm 854$ & $i$ & 1 \\
\hline \multirow[t]{6}{*}{ PG1444+407 } & $-27 \pm 19$ & $u$ & 1 \\
\hline & $46 \pm 119$ & $g$ & 1 \\
\hline & $-135 \pm 161$ & $r$ & 1 \\
\hline & $292 \pm 334$ & $i$ & 1 \\
\hline & $57 \pm 130$ & F606W & 2 \\
\hline & $49 \pm 79$ & F606W & 3 \\
\hline \multirow[t]{7}{*}{ PG1545+210 } & $133 \pm 38$ & $u$ & 1 \\
\hline & $104 \pm 120$ & $g$ & 1 \\
\hline & $0^{3}$ & $r$ & 1 \\
\hline & $1643 \pm 404$ & $i$ & 1 \\
\hline & $206 \pm 199$ & F606W & 2 \\
\hline & $242 \pm 79$ & F606W & 3 \\
\hline & $236 \pm 120$ & $r$ & 4 \\
\hline \multirow[t]{4}{*}{ PG1612+261 } & $56 \pm 27$ & $u$ & 1 \\
\hline & $354 \pm 237$ & & 1 \\
\hline & $-116 \pm 397$ & $r$ & 1 \\
\hline & $-935 \pm 727$ & $i$ & 1 \\
\hline \multirow[t]{3}{*}{ Q2141+175 } & $370 \pm 221$ & $g$ & 1 \\
\hline & $991 \pm 355$ & $r$ & 1 \\
\hline & $112 \pm 169$ & F675W & 2 \\
\hline
\end{tabular}

${ }^{1}$ Here we quote results for $\gamma=1.77$ and a radius of $0.5 h^{-1} \mathrm{Mpc}$; Literature values have been scaled by $\left(h^{-1}\right)^{1.77}$, but are otherwise uncorrected for different cosmologies.

2 References: (1)this work; (2) McLure \& Dunlop (2001b); (3) Finn et al. (2001); (4) Yee \& Green $(1984)$

${ }^{3} N_{\mathrm{t}}=N_{\mathrm{b}}$

be a rich cluster environment, and clusters with Abell classes 0,1 and 2 show values of $\sim 350$, $\sim 650$, and $\sim 950$ respectively, according to Yates et al. (1989). McLure \& Dunlop (2001b) suggest a classification based on that of Yee \& López-Cruz (1999), 


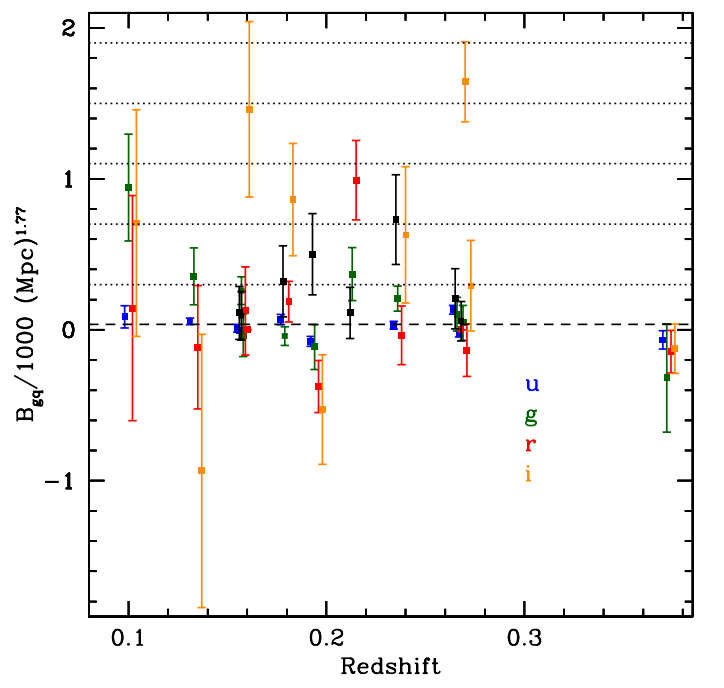

FIG. 11.- B $_{g q}$ versus quasar redshift for all filters. Points have been offset slightly in $\mathrm{z}$ for clarity. Black points show values from the literature listed in Table 7 Dotted lines, bottom to top, mark the $\mathrm{B}_{g q}$ values for Abell classes 0-4 (Yee \& López-Cruz 1999) and the dashed line marks the galaxy-galaxy correlation value from Davis \& Peebles (1983).

where $\mathrm{B}_{g q}=(300,700,1100,1500,1900,2300)$ corresponds to Abell class $(0,1,2,3,4,5)$ respectively. Given the large statistical uncertainties in the $\mathrm{B}_{g q}$ values, we find results marginally greater than 500 in only a few cases, for PG1307+085 and PG1545+210 in the $i$ band, $1461 \pm 778$ and $1643 \pm 404$, respectively, for PG1404+226 in the $g$ band, $942 \pm 522$, and for Q2141+175 in the $\mathrm{r}$ band, $991 \pm 355$. The radio loud AGN PG1545+210 is known to reside in an Abell class 1 environment (Oemler et al. 1972), though our $i$ band $\mathrm{B}_{g q}$ value is consistent with Abell class 3. The $\mathrm{B}_{g q}$ values for this field in $g$ and $r$ are consistent with $\mathrm{B}_{g g}$, and in $u$ we find only $133 \pm 38$, which is significantly larger than $\mathrm{B}_{g g}$, but far smaller than the $i$ band value. PG1307+085 has a foreground galaxy cluster in its field at a close projected separation from the quasar and with a similar redshift, $z_{\text {clus }}=0.14$ versus $z_{q}=0.155$; and PG1404+226 has a background cluster within the $13.1^{\prime}$ radius corresponding to $0.5 h^{-1}$ Mpc. Q2141+175 is the only other radio loud AGN in our sample and while it has no known cluster at its redshift or in projection, it is a less well-studied field than many of the others. Thus the $\mathrm{B}_{g q}$ for these other fields may be influenced by foreground, background, or even proximate galaxy clusters, but only in select bands.

There are a handful of other fields that show a significant, positive signal in $\mathrm{B}_{g q}$ in one or more bands, and these are discussed in Appendix A. One field, PG0923+201, shows negative values of $\mathrm{B}_{g q}$ in all bands, with two $(u, r)$ at $>2 \sigma$. As we discuss in Appendix $\mathrm{A}$ this well-studied field has a compact group consistent with the redshift of the AGN and with a center only 23 $h^{-1} \mathrm{kpc}$ from the position of the AGN, making this result an intriguing one. Three other fields, HS0624+690, MRK586, and PG1444+407 show $\mathrm{B}_{g q}$ values in all filters that are either consistent with or significantly less than $\mathrm{B}_{g g}$. HS0624+690 is the only quasar in our sample, and with $z=0.370$, our limiting magnitudes in $u$ and $g$ allow us to reach only $\sim M^{*}+0.7$ and $\sim M^{*}+0.1$, respectively.
TABLE 8

Galaxy-Galaxy Clustering Clustering in 90Prime QSO Fields ${ }^{1}$

\begin{tabular}{lccl}
\hline \hline Filter & $\gamma$ & $A_{\text {gg }}$ & $\chi_{\nu}$ \\
\hline$u^{2}$ & $1.51 \pm 0.02$ & $0.13 \pm 0.05$ & 2.9 \\
$g^{2}$ & $1.50 \pm 0.03$ & $0.26 \pm 0.08$ & 10.9 \\
$r$ & $1.59 \pm 0.03$ & $0.18 \pm 0.04$ & 12.5 \\
$i$ & $1.53 \pm 0.02$ & $0.18 \pm 0.03$ & 27.1 \\
& & \\
\hline 1 & & & \\
2 & & \\
fiers masked &
\end{tabular}

Two other AGN in our sample, PG1116+215 and PG1612+261 lie near galaxy clusters, though their redshifts and position on the sky place them near the peripheries of those clusters. The $\mathrm{B}_{g q}$ values for these fields give no indication of a cluster association: for $\mathrm{PG} 1116+215$, all the $\mathrm{B}_{g q}$ values are consistent with $\mathrm{B}_{g g}$, except the anomalously high value we find for the $i$ band, $863 \pm 461$. For PG1612+261, the only filter showing a $\mathrm{B}_{g q}$ marginally larger than $\mathrm{B}_{g g}$ is the $g$ band, for which we find $354 \pm 237$, Abell class 0 .

\subsubsection{Correlation Functions}

We show the results for the normalization, $A_{\mathrm{gg}}$, and slope, $\gamma$ of the galaxy autocorrelation function in Figure 12 and list the results in Table 8. These calculations were performed for Sample 2. For the $u$ and $g$ filters, we use only chips 1 and 4 as chips 2 and 3 added extra structure due to masking. The $r$ and $i$ filters typically are less affected by this. The use of all chips for $u$ and $g$ gives similar results, but a poorer fit to the form of the autocorrelation function. We avoid separations less than $10^{\prime \prime}$ to mitigate against blending, and because we are restricted to single chips, we calculate the correlation function to a maximum separation of $10^{\prime}$. The errors on the parameters are derived from the variance in that parameter from 100 jackknife samples of the dataset, where the sampling with replacement was done one field/filter at a time.

In our calculations of the galaxy-quasar crosscorrelations, we consider quasars in 90Prime fields other than the target quasars themselves, as any clustering may also be present for these objects as well. As discussed above, the galaxy counts in our images are very sparse at $z>1$ and so galaxies associated with clusters, groups, or quasars at these redshifts are generally not of concern to us here. AGN with $z<0.5$ found in the 90Prime frames are listed in Table 9 and the resulting quasar redshift distributions in each filter are shown in Figure 13. The absolute magnitudes relative to $\mathrm{M}^{*}$ reached for $z=0.5$ galaxies in Samples 1 and 2 are listed in Table 6

We list the results for the normalization $\mathrm{A}_{g q}$ of the galaxy-quasar cross-correlation function in Table 10 for both Samples 1 and 2, where the latter correspond to deeper magnitude limits and fainter galaxy samples. For these solutions, we hold the slope $\gamma$ to be constant at 1.8 , the commonly accepted value and 1.5, corresponding to the slope we found from the 90Prime data for the galaxygalaxy clustering on $10^{\prime}$ scales. This difference in slope changes the normalization at only the $\sim 1 \sigma$ level. The 


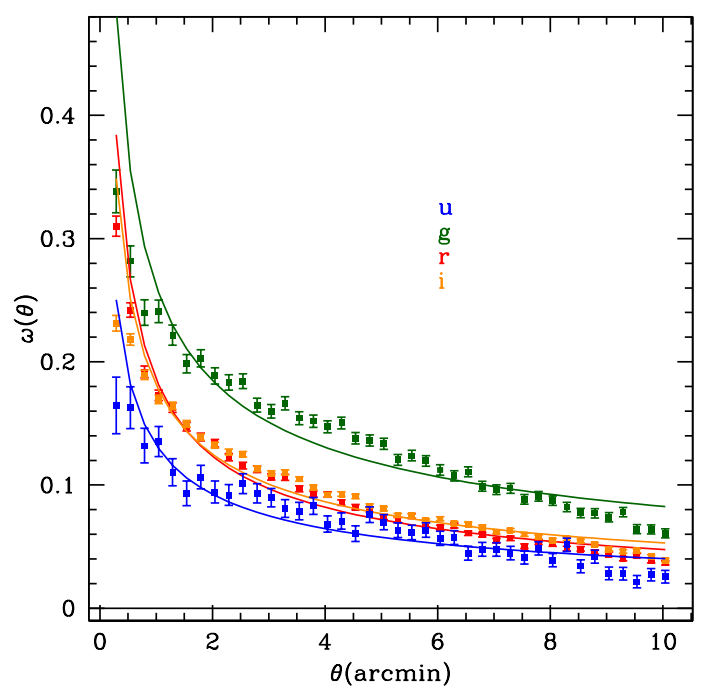

FIG. 12.- Galaxy-galaxy angular correlation function in $u, g, r, i$ filters for 90Prime fields.

TABLE 9

Other $z<0.5$ QUASARS IN 90PRIME FieldS

\begin{tabular}{|c|c|c|}
\hline QSO & $\mathrm{z}$ & Filters \\
\hline \multicolumn{3}{|l|}{ PG0844+349 } \\
\hline SDSS J084731.77+351416.4 & 0.237 & $u, r$ \\
\hline 2MASX J08503620+3455231 & 0.144 & $u, r$ \\
\hline \multicolumn{3}{|l|}{ PG0923+201 } \\
\hline SDSS J092507.72+203540.9 & 0.472 & $g, i, r$ \\
\hline SDSS J092525.16+202139.0 & 0.460 & $u, g, r$ \\
\hline SDSS J092536.08+201649.5 & 0.228 & \\
\hline \multicolumn{3}{|l|}{ PG1307+085 } \\
\hline Q87GBBWE91 1307+0843 & & \\
\hline SDSS J131155.76+085340.9 & 0.469 & $u, g, r, i$ \\
\hline \multicolumn{3}{|l|}{ PG1444+407 } \\
\hline SDSS J144618.08+412003.0 & 0.268 & $u, g, r, i$ \\
\hline \multicolumn{3}{|l|}{ PG1545+210 } \\
\hline SDSS J154749.70+205056.5 & 0.265 & $u, g, r$ \\
\hline SDSS J154750.71+210351.1 & 0.296 & $u, g, r$ \\
\hline SDSS J155014.81+212431.5 & 0.479 & \\
\hline SDSS J155046.30+205803.1 & 0.401 & $u, g, r, i$ \\
\hline 2MASS J15505930+2128088 & 0.372 & $u, r$ \\
\hline \multicolumn{3}{|l|}{ PG1612+261 } \\
\hline HB89 $1612+266$ & 0.395 & $u, g, r, i$ \\
\hline
\end{tabular}

results for both slopes are plotted in Figure 14

Croom \& Shanks (1999) found no galaxy-quasar correlation in their sample of $\sim 150$ optically and X-ray selected quasars with $z=1-1.5$ and $b_{J}<23$ galaxies in five deep AAT plate fields. In fact, their data showed a weak anti-correlation between these two populations. At the the lower redshifts of our sample, we see significant correlations only in the $u$ band. For Sample 1, there is also a significant positive correlation on the $r$ band, but this does not hold with the inclusion of fainter galaxies in Sample 2. In fact in all bands except the $i$ band, the correlation is significantly smaller for Sample 2, and we also see weak anti-correlations in several cases. In $i$, the normalizations are all consistent with zero for both Samples 1 and 2 .

In Table 10, we also tabulate the correlation length for galaxy-quasar clustering, $r_{0}$, found from Limber's equa-

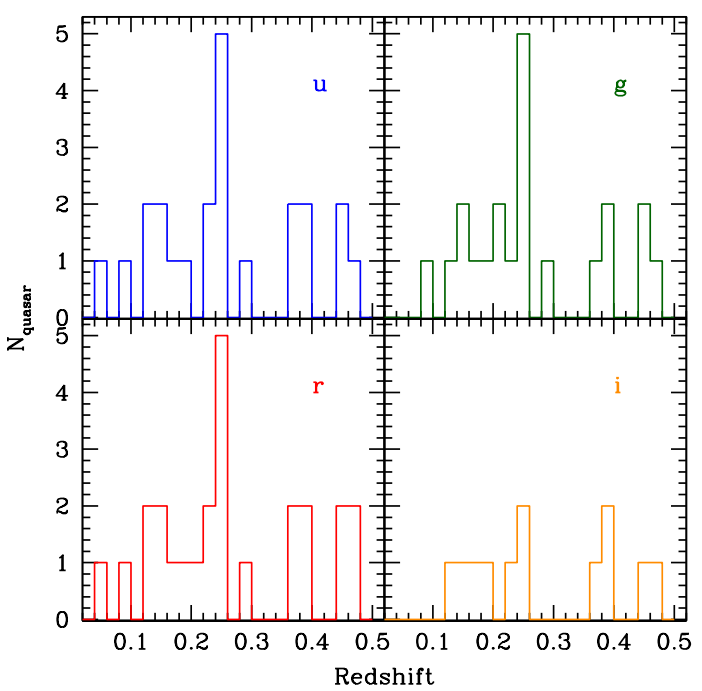

FIG. 13.- Redshift distributions of all quasars in the 90Prime fields

TABLE 10

Galaxy-Quasar Clustering in 90Prime QSO FIELDS ${ }^{1}$

\begin{tabular}{lccc}
\hline \hline Filter & $A_{\mathrm{gq}}$ & $\chi_{\nu}$ & $\mathrm{r}_{0} \mathrm{~h}^{-1} \mathrm{Mpc}^{2}$ \\
\hline \multicolumn{4}{c}{ Sample $1, \gamma=1.8$} \\
\hline$u$ & $0.49_{-0.14}^{+0.15}$ & 0.39 & 8.07 \\
$g$ & $0.23_{-0.14}^{+0.16}$ & 0.69 & 5.36 \\
$r$ & $0.21_{-0.07}^{+0.08}$ & 0.70 & 6.11 \\
$i$ & $-0.03 \pm 0.07$ & 1.66 & $\ldots$ \\
\hline \multicolumn{4}{c}{ Sample $1, \gamma=1.5$} \\
\hline$u$ & $0.61_{-0.19}^{+0.21}$ & 0.38 & 16.9 \\
$g$ & $0.34_{-0.19}^{+0.22}$ & 0.65 & 11.64 \\
$r$ & $0.28 \pm 0.10$ & 0.65 & 12.3 \\
$i$ & $-0.0027_{-0.09}^{+0.10}$ & 1.68 & $\ldots$ \\
\hline \multicolumn{5}{c}{ Sample $2, \gamma=1.8$} \\
\hline$u$ & $0.40_{-0.11}^{+0.12}$ & 0.61 & 7.76 \\
$g$ & $-0.04 \pm 0.09$ & 1.03 & $\ldots$ \\
$r$ & $-0.05 \pm 0.05$ & 1.57 & $\ldots$ \\
$i$ & $0.004 \pm 0.05$ & 1.44 & 0.90 \\
\hline \multicolumn{5}{c}{ Sample $2, \gamma=1.5$} \\
\hline$u$ & $0.51_{-0.15}^{+0.16}$ & 0.57 & 16.2 \\
$g$ & $-0.03_{-0.11}^{+0.12}$ & 1.04 & $\ldots$ \\
$r$ & $-0.06_{-0.06}^{+0.07}$ & 1.58 & $\ldots$ \\
$i$ & $0.02 \pm 0.07$ & 1.44 & 2.95 \\
5
\end{tabular}

${ }^{1} 0.1667^{\prime}-10.1667^{\prime}$

$2 \epsilon=0$
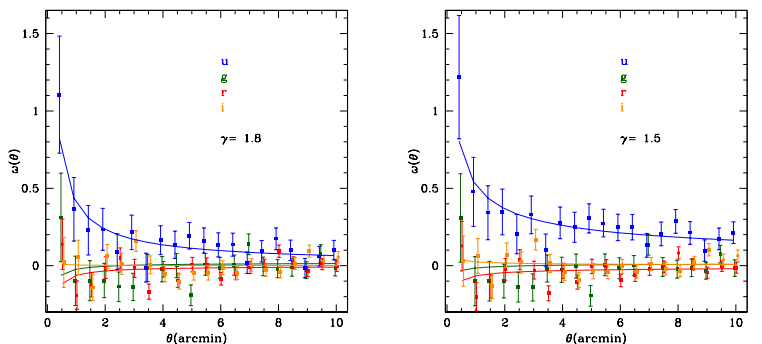

FIG. 14.- Quasar-galaxy angular correlation functions in $u, g, r, i$ filters for 90Prime fields for $\gamma=1.8$ and $\gamma=1.5$. Points are offset in $\mathrm{x}$ and $\mathrm{g}$ fit curve is offset in $\mathrm{y}$ by +0.02 for clarity. 


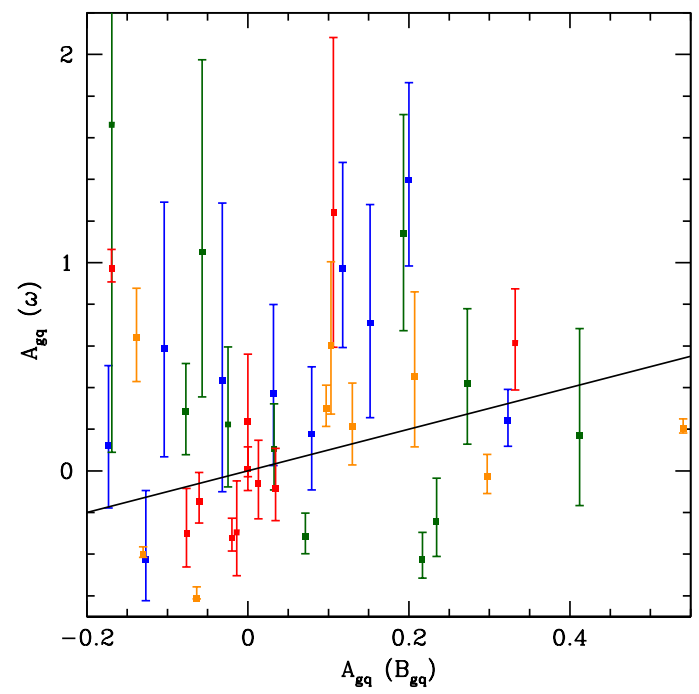

FIG. 15.- Comparison of the two methods for calculating the angular covariance amplitude, $\mathrm{A}_{g q}$ from Equation 7 and Equation 13 both with $\gamma=1.8$.

tion, Equation 9, and the galaxy and quasar redshift distributions plotted in Figures 10 and 13. This is undefined for negative values of $\mathrm{A}_{g q}$, so no correlation length values are reported in Table 10 in these cases. The values we find range from $0.9 h^{-1} \mathrm{Mpc}$ for Sample 2 in the $i$ band with $\gamma=1.8$ to $\sim 16 h^{-1}$ Mpc for both Samples 1 and 2 in the $u$ band with $\gamma=1.5$. These results are generally within the range found for $0.7<z<1.4$ quasars in the DEEP2 survey by Coil et al. (2007), $0.1 h^{-1} \lesssim r_{0} \lesssim$ $10 h^{-1}$ Mpc. Brown et al. (2001) studied a population of galaxy fields around a sample of moderate redshift quasars with $z=0.2-0.7$ and also find a weak correlation, though a stronger one for red galaxies $\left(r_{0} \sim 7\right.$ $\left.h^{-1} \mathrm{Mpc}, \gamma \sim 1.9\right)$ than for blue galaxies $\left(r_{0} \lesssim 4 h^{-1}\right.$ $\mathrm{Mpc}$ ). These authors provide a summary of results to that time in their Table 1. The value of the correlation length ranges from $\sim 5-8 h^{-1} \mathrm{Mpc}$ for samples of radio quiet quasars with similar redshift but shallower depth than our current 90Prime study to $\sim 12-17 h^{-1} \mathrm{Mpc}$ for fields around radio loud quasars, comparable to our result for the $u$ band in the 90Prime sample. Below where we discuss trends of galaxy-quasar clustering with quasar properties, we look at the question of whether the two radio loud objects in our sample show significantly greater galaxy clustering than the radio quiet objects.

In our two methods of investigating galaxy-quasar correlations, outlined in Sections 4.2 and 4.2 .2 we calculate the angular covariance amplitude, $\mathrm{A}_{g q}$ in two different ways, one involving a comparison with a set of random galaxy positions and one using the expectation from an integral of the galaxy luminosity function in the relevant band. In Figure 15, we compare the values obtained from these two methods for $\gamma=1.8$. We note that the uncertainties in the fitted $\omega(\theta)$ for a single field/filter can be large, and the offset for any one field/filter can be large, ranging from -0.6 to +1.8 , but the mean offset in these values is 0.22 with a standard deviation of 0.55 . As has been noted in previous work, clustering statistics like $\mathrm{B}_{g q}$ can be sensitive to the methodology used to calculate it, the choice of luminosity function and control fields. We
TABLE 11

Sample Quasar Properties

\begin{tabular}{lcccll}
\hline \hline \multicolumn{1}{c}{ QSO } & $\mathrm{M}_{r}$ & $\log \left(\mathrm{M}_{\mathrm{BH}}\right)$ & Refn. $^{1}$ & $\begin{array}{r}{[\mathrm{O} \text { III }] \text { Luminosity }} \\
\mathrm{erg} / \mathrm{s} \times 10^{42}\end{array}$ & Refn. $^{2}$ \\
\hline MRK586 & -23.4 & 8.34 & 1 & 3.50 & 1 \\
HS0624+690 & -26.0 & 9.69 & 2 & $\ldots$ & $\ldots$ \\
PG0844+349 & -22.4 & 7.96 & 3 & $\ldots$ & $\ldots$ \\
PG0923+201 & -23.7 & 8.00 & 4 & 0.99 & 3 \\
PG0953+414 & -24.7 & 8.44 & 3 & 1.90 & 3 \\
PG1116+215 & -24.6 & 8.52 & 4 & 2.60 & 2 \\
PG1307+085 & -23.2 & 8.64 & 3 & 3.17 & 2 \\
PG1404+226 & -21.8 & 6.88 & 4 & 0.38 & 2 \\
PG1444+407 & -24.1 & 8.28 & 4 & $2.22^{3}$ & 2 \\
PG1545+210 & -24.4 & 9.31 & 4 & 4.93 & 1 \\
PG1612+261 & -22.6 & 8.05 & 4 & $6.68^{4}$ & \\
Q2141+175 & -23.6 & 8.74 & 1 & 2.80 & \\
\hline
\end{tabular}

1 References: (1) McLure \& Dunlop (2001a); (2) Labita et al. (2006); (3) Peterson et al. (2004); (4) Vestergaard \& Peterson (2006)

2 References: (1) Ho \& Kim (2009); (2) This study; (3) Shang et al. (2007) 3 Shang et al. (2007) find 0.22

${ }^{4}$ Sum of two components FWHM $=420 \mathrm{~km} \mathrm{~s}^{-1}$ and $1000 \mathrm{~km} \mathrm{~s}^{-1}$ with fluxes 4.75 and 1.93 respectively

therefore concentrate less on the absolute values of this parameter than on investigating any trends in the data, discussed in the following section.

\subsection{Trends in Clustering Parameters \\ 5.2.1. Correlation Amplitude}

We investigate trends of galaxy-quasar clustering with quasar absolute $r$ magnitude, black hole mass, and [O III] line luminosity, as an indicator of AGN activity (Kauffmann et al. 2004). These properties are listed in Table 11] The values of $\mathrm{M}_{r}$ are derived from the $r$ listed in the SDSS DR9 catalog, with Galactic extinction calculated using the reddening values from Schlafly \& Finkbeiner (2011) and the O’Donnell (1994) reddening law. We also applied a K-correction, calculated from the QSO composite spectrum of Vanden Berk et al. (2001) and the SDSS filter response curves. We collected black hole mass and [O III] $\lambda 5007$ line luminosity measurements from the literature and list them in Table [11. Where the [O III] line luminosities do not exist in the literature, we downloaded the SDSS DR10 spectra for these objects and fit the $\mathrm{H} \beta$ [O III] $\lambda 4959,5007$ complex using the IRAF task specfit and list the resulting value in Table 11

Plots of these quasar properties with $\mathrm{B}_{g q}$ parameter are shown in Figures 16, 18, but in no case do we find a significant trend of this clustering measure with any of the quasar properties.

\subsubsection{Correlation Functions}

We also investigate trends of the galaxy-quasar angular correlation function with quasar luminosity, black hole mass, and [O III] line luminosity by using the relative bias with respect to galaxies, $b_{g q}=\left(\omega_{g q} / \omega_{g g}\right)^{1 / 2}$. Overall, we find $b_{g q}=(3.08 \pm 0.51,1.49 \pm 0.53,1.48 \pm 0.29,0.95 \pm$ 1.49) for $(u, g, r, i)$ for cross- and autocorrelation function solutions with fixed $\gamma=1.5$. The bias is only significantly greater than unity for the $u$ band and decreases with increasing wavelength, perhaps reflecting a trend seen for SDSS Sy 2 galaxies to lie in bluer environments than 


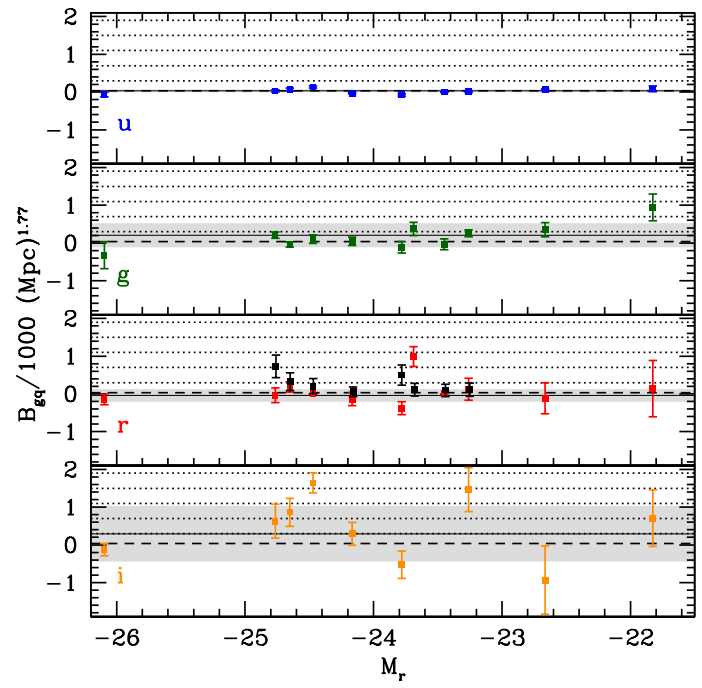

FIG. 16.- $\mathrm{B}_{g q}$ in each filter versus quasar absolute $\mathrm{r}$ magnitude. Solid lines and shaded regions mark the mean and dispersion among all the values in a given filter, dotted lines show the expected correlation amplitude for Abell classes 0-4 (Yee \& López-Cruz 1999), and the dashed line marks the galaxy-galaxy correlation value from Davis \& Peebles (1983). Black points show values from the literature listed in Table 7

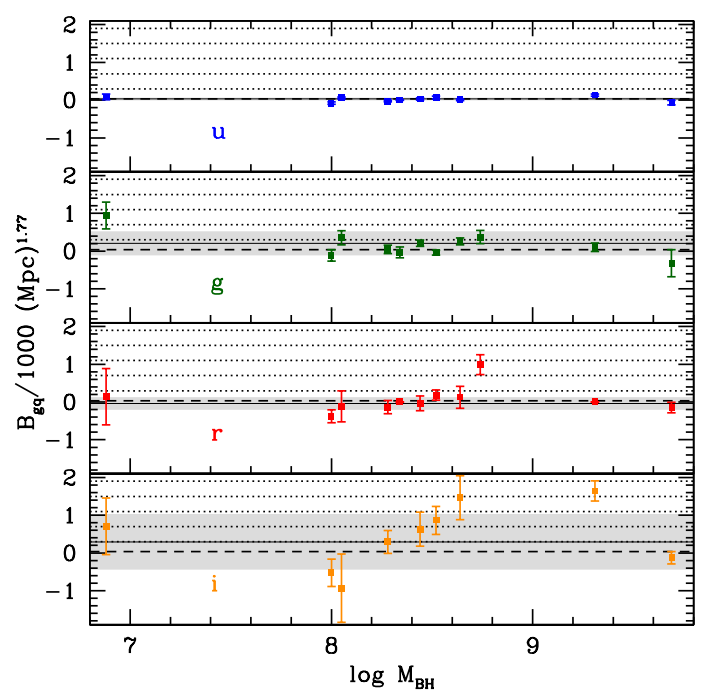

FIG. 17.- As in Figure 16 but for $\mathrm{B}_{g q}$ in each filter versus quasar black hole mass

normal galaxies (Coldwell et al. 2014). This runs counter to a trend in the mean $\mathrm{B}_{g q}$ values quoted above, for which the $u$ band values are lowest and those in $i$ are the largest. This is driven by the large $i$ band $\mathrm{B}_{g q}$ values found for PG1545+201 and PG1307+085, the former being a radio loud quasar. That trend is less apparent for the radio quiet objects alone, and without PG1307+085 as well, the average $i$ band $\mathrm{B}_{g q}$ value drops to $129 \pm 238$.

The values of $\omega$ for each field and filter individually are listed in Table 12. For each field/filter where both the cross- and autocorrelation values are positive in the innermost bin, $\omega<1.167^{\prime}$, we find no correlations in $b_{g q}$ with any of the quasar properties discussed above. Most fields for which both $\omega_{g q}$ and $\omega_{g g}$ within $1.167^{\prime}$

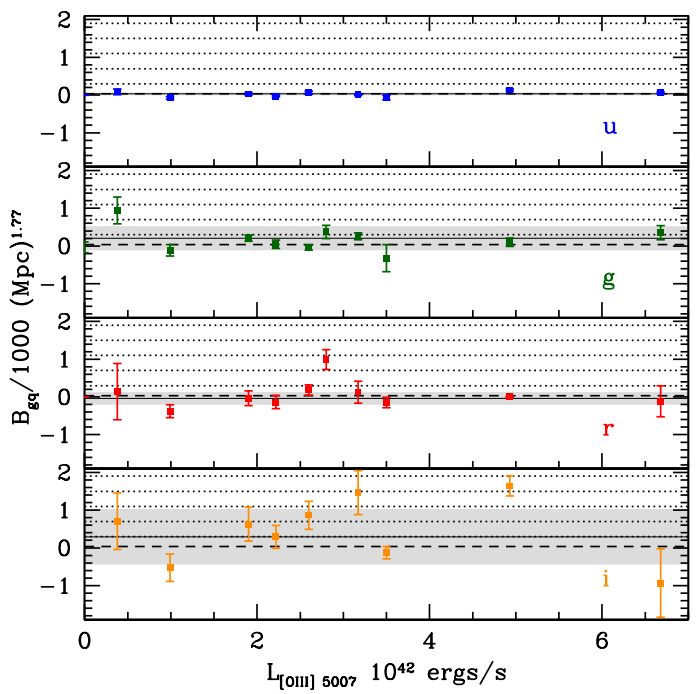

Fig. 18.- As in Figure 16 but for $\mathrm{B}_{g q}$ in each filter versus quasar [O III] luminosity

are positive show $b_{g q}>1$, but only a handful at $\gtrsim 2 \sigma$ : PG0844+349 and PG1612+261 in $u$, HS0624+690 in $g$, PG0923+201 and PG1116+215 in $r$, and PG1404+226 in $i$. For PG0844+349 and PG1612+261 the filters with $b_{g q}>1$ are the only filters with $\omega_{g q}>0$, for which $b_{g q}$ is defined. That the $u$ filters would show a large positive relative bias while all others show a quasar-galaxy anticorrelation may be indicative of bluer environments, although this does not hold for the HS0624+690 field, for which the $b_{g q}$ in $g$ is $1.31 \pm 0.094$ but the values of $\omega_{g q}$ are negative in all other filters, including $u$. A few fields show $b_{g q}>1$ consistently in all filters (PG0923+201, PG1116+215) or in all filters but one: PG1307+085 (i), PG1404+226 $(r)$. The bias seen in the $u$ band for the radio loud quasar PG1545+210 is negative, and not significantly greater than unity in any filter. Similarly, for the other radio loud AGN in our sample, Q2141+175, only $g$ and $r$ band data are available and neither shows a large bias. In $g, \omega_{g q}<0$, and in $r b_{g q}=0.66 \pm 0.75$.

\section{DISCUSSION}

A somewhat contradictory picture emerges when we compare the results of the correlation function analysis to the calculated $\mathrm{B}_{g q}$ statistics. The fields that show the largest values of $\mathrm{B}_{g q}, \mathrm{PG} 1307+085, \mathrm{PG} 1545+210$, PG0953+414 in the $i$ band, PG1404+226 in $g$, and $\mathrm{Q} 2141+175$ in $r$, all show relative biases that are consistent with unity. The PG0923+201 field showed an anticlustering $\mathrm{B}_{g q}$ signal in $u$ and $r$, but both filters give $b_{g q}>1$, with the $r$ band significant at $\sim 3 \sigma$. We note that, in addition to a luminosity function dependence, the $\mathrm{B}_{g q}$ analysis probes a larger spatial scale than the relative bias calculation within $1.167^{\prime}$, corresponding to $\sim 10-40 h^{-1} \mathrm{kpc}$ over the redshift range of the AGN in our sample.

Overall, as noted above in Section 5.1.1, the mean values of the correlation amplitude for $0.5 h^{-1} \mathrm{Mpc}$ scale clustering, while all consistent within the uncertainties with galaxy-galaxy clustering, show the largest signal in the $i$ band, $\mathrm{B}_{g q}=295 \pm 260$. The correlation function analysis, on the other hand, probing $\sim 10-40 h^{-1} \mathrm{kpc}$ scales 
TABLE 12

Galaxy-GalaXy AND Galaxy-QuASAR Correlations FOR EACH 90PRIME Field ${ }^{1}$

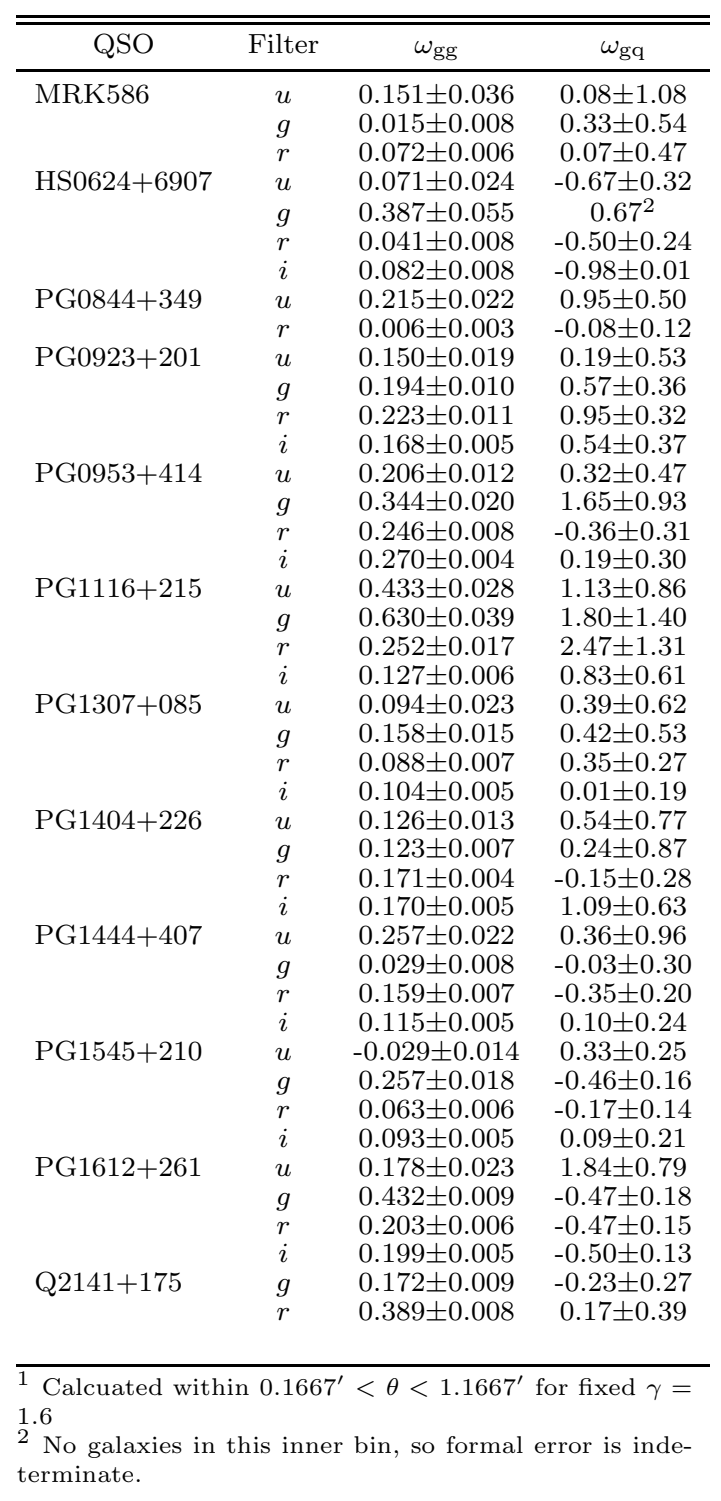

shows the largest bias for quasar galaxy clustering in the $u$ band, with galaxy clustering around quasars a factor of 3 larger than the galaxy autocorrelation signal at these scales. This suggests that different galaxy types may cluster around quasars on different scales. Some earlier studies have seen clustering signals at small scales and differences in galaxy populations near quasars. In a future paper, we will use the multi-band data to calculate photometric redshifts for the galaxies in our sample, and investigate trends in the clustering parameters with galaxy types determined with these redshifts.

The basic trends in our results are consistent with earlier findings that quasars cluster like $\mathrm{L}^{*}$ galaxies, and indeed like galaxies up to 2 magnitudes fainter than $\mathrm{L}^{*}$. We also confirm that there are no significant trends with quasar luminosity (Finn et al. 2001; Serber et al. 2006; Coil et al. 2007), though unlike Serber et al. (2006), we also find that the lack of luminosity dependence holds at small as well as large spatial scales. In our sample of broad line AGN, we do not find the trend for AGN with lower [O III] luminosities to have larger clustering signals as seen in SDSS narrow line AGN (Wake et al. 2004).

Thus, the mechanism driving accretion onto the central black holes in these objects is not imprinted on their large scale galaxy environments. This may be due to a nonmerger mode in fueling the central black holes or to a delay in the onset of nuclear activity from the time of the initial galaxy interactions which triggered its fueling.

J. S. and A. R. acknowledge the support of the National Science Foundation (AST-0952923).

This research has made use of the NASA/IPAC Extragalactic Database (NED) which is operated by the Jet Propulsion Laboratory, California Institute of Technology, under contract with the National Aeronautics and Space Administration.

Funding for the SDSS and SDSS-II has been provided by the Alfred P. Sloan Foundation, the Participating Institutions, the National Science Foundation, the U.S. Department of Energy, the National Aeronautics and Space Administration, the Japanese Monbukagakusho, the Max Planck Society, and the Higher Education Funding Council for England. The SDSS Web Site is http://www.sdss.org/.

The SDSS is managed by the Astrophysical Research Consortium for the Participating Institutions. The Participating Institutions are the American Museum of Natural History, Astrophysical Institute Potsdam, University of Basel, University of Cambridge, Case Western Reserve University, University of Chicago, Drexel University, Fermilab, the Institute for Advanced Study, the Japan Participation Group, Johns Hopkins University, the Joint Institute for Nuclear Astrophysics, the Kavli Institute for Particle Astrophysics and Cosmology, the Korean Scientist Group, the Chinese Academy of Sciences (LAMOST), Los Alamos National Laboratory, the Max-Planck-Institute for Astronomy (MPIA), the MaxPlanck-Institute for Astrophysics (MPA), New Mexico State University, Ohio State University, University of Pittsburgh, University of Portsmouth, Princeton University, the United States Naval Observatory, and the University of Washington.

Facilities: Bok

\section{APPENDIX}

\section{NOTES ON INDIVIDUAL OBJECTS}

In this section, we outline notes on each of our 90Prime fields, based on previous studies of the quasar fields and host galaxies, searches for nearby objects in the NASA Extragalactic Database (NED), and the results of our calculations of the correlation amplitude, $\mathrm{B}_{g q}$. We refer the reader to Table 7 and Figures 11 18. 


$$
\operatorname{Mrk} 586, z=0.155
$$

The field of this radio quiet object has been imaged previously in narrow band [O III] by Stockton \& MacKenty (1987) and in the optical (B, $g, i$ filters) by Kirhakos et al. (1994) using the Palomar $1.5 \mathrm{~m}$ (image sizes $\left.\sim 3^{\prime} \times 3^{\prime}\right)$ who note four galaxies in this field, including a large disk galaxy (NAB 0205+02: 06) at a projected separation of $15.6 h^{-1}$ kpc.

The quasar host was studied by (Bahcall et al. 1997) using the HST/ Wide Field/Planetary Camera-2 (WFPC2) and the F606W filter. The host is disk-like and the AGN has a companion with jet-like structure at a projected distance of $23 h^{-1}$ kpc (Stockton \& MacKenty 1987; Bahcall et al. 1997). There is no evidence of tidal features to suggest a direct interaction.

A NED search reveals $\sim 17$ galaxy clusters, groups, or candidates, within $50^{\prime}$ of the quasar. The cluster with the smallest angular separation from the quasar $\left(7.7^{\prime}\right)$ is NSCS J020723+024645, which lies at $z=0.37$.

The $\mathrm{B}_{g q}$ we calculate from the 90Prime $r$ band image is identically zero because the number of galaxies detected at $5 \sigma$ within $0.5 \mathrm{~h}^{-1} \mathrm{Mpc}$ of the quasar exactly equals the median number found in 871 control fields. This is consistent with the values found by both McLure \& Dunlop (2001b) and Finn et al. (2001) from the HST/WFPC2 images in the F606W filter, $93 \pm 161$ and 13 \pm 82 , respectively, and with the values calculated from the 90Prime $u$ and $g$ band images, $-3 \pm 6$ and $-36 \pm 137$, respectively. We have no 90Prime data of this field in the $i$ band.

$$
\text { HS0624+690, } z=0.370, Q S O
$$

The host of this radio quiet quasar is an elliptical galaxy (Floyd et al. 2004). Kirhakos et al. (1994) identify six galaxies in their ground-based optical images within the $\sim 3^{\prime} \times 3^{\prime}$ frame. From NED, we find that within $50^{\prime}$ of the quasar, there is one known galaxy cluster, Abell 0557 (redshift unknown), at 8.03' separation from the quasar.

All the $\mathrm{B}_{g q}$ values we calculate for this field are less than zero, indicating an underdensity of galaxies within $0.5 h^{-1}$ $\mathrm{Mpc}$ of the quasar, however, none of these underdensities is significantly less than $\mathrm{B}_{g g}$ at $>2 \sigma$. We conclude that the field around this object is consistent with the field. A caveat to this is that HS0624+690 is the the highest redshift AGN in our sample, and the limiting magnitudes of the $u$ and $g$ coadded images are not significantly fainter than $\mathrm{M}^{*}$ at the quasar redshift, $\mathrm{M}^{*}+0.7$ and $\mathrm{M}^{*}+0.1$, respectively, so we are not reaching as far down the luminosity function for this field as the others in the sample.

$$
P G 0844+349, z=0.064
$$

This radio quiet object has a host with bulge+disk structure, showing no bar, but some evidence of morphological disturbance from deep H-band imaging (Guyon et al. 2006; Veilleux et al. 2009). This object qualifies as having a bright interacting companion $\left(\mathrm{H}\right.$ band luminosity greater than $1 / 10 \mathrm{~L}_{*}$ and projected distance within $24 h^{-1} \mathrm{kpc}$ McLeod \& Rieke (1994b), though it is not likely currently undergoing a strong interaction since Veilleux et al. (2009) classify this object as a post-merger object with a single nucleus. Also, from the [O II] measurement of Wilkes et al. (1999), $\mathrm{Ho}(2005)$ estimates a star formation rate of the host galaxy of less than $1.6 \mathrm{M}_{\odot} \mathrm{yr}^{-1}$. Within a $50^{\prime}$ radius around this quasar, NED returns 58 galaxy clusters, groups, or candidates.

$$
P G 0923+201, z=0.192
$$

The host of this radio quiet AGN is an elliptical galaxy with no evidence of a bar or of morphological disturbance from an interaction with a companion (Dunlop et al. 2003; Guyon et al. 2006; Veilleux et al. 2009). Previous authors have note the presence of two bright galaxies within $16 h^{-1} \mathrm{kpc}$ of the quasar and have suggested it is a member of a small group (Heckman et al. 1984; McLeod \& Rieke 1994b; Bahcall et al. 1997). Indeed, the compact group SDSSCGB 30540 ( $z=0.19)$ identified by McConnachie et al. (2009) in the SDSS DR6 has a redshift consistent with that of PG0923+201. While the quasar is not listed as a group member in their catalog, it has a separation of $\sim 23$ $h^{-1} \mathrm{kpc}$ from the group center. There are $\sim 45$ other galaxy clusters or groups within $50^{\prime}$ of this quasar.

Our $\mathrm{B}_{a q}$ values from the $r$ and $i$ band frames, $-377 \pm 130$ and $-529 \pm 291$, are inconsistent with those of McLure \& Dunlop (2001b) and Finn et al. (2001) at the 3.5-4 $\sigma$ level. These authors find moderately significant overdensities in this field while we see underdensities in all filters, of increasing magnitude from $u$ to $i$. This underdensity is surprising if the quasar is in fact associated with SDSSCGB 30540.

$$
P G 0953+414, z=0.234
$$

Dunlop et al. (2003) classify the host galaxy of this radio quiet AGN as an elliptical, while others (Bahcall et al. 1997; Guvon et al. 2006) have found it to show disk-like structure but to be too faint to classify reliably. Kirhakos et al. (1994) find 22 likely galaxies in their optical images described above in Sec. A.1.

There are 54 galaxy groups, clusters or candidates within $50^{\prime}$ of this quasar, one of which, GMBCG J149.20633+41.27869, has a projected separation of only $1.455^{\prime}$, though it lies well behind PG0953+414, at $z=0.362$. A second cluster, WHL J095714.9+411700, is separated by $4.532^{\prime}$ from the quasar and has a redshift $z=0.446$.

Our $i$ band result for $\mathrm{B}_{g q}$ is consistent with the result of McLure \& Dunlop (2001b) using HST/WFPC2 F675W. Both values are large, $\sim 600-700$, but with large statistical uncertainty, $\sim 300-400$. The $g$ band value for this field, $207 \pm 101$, is one of $\sim 1 / 4$ of the $\mathrm{B}_{g q}$ results that are significantly greater than $\mathrm{B}_{g g}$, while the $\mathrm{B}_{q q}$ result from the 90Prime $r$ band image is negative but consistent with $\mathrm{B}_{g g}$, and consistent with the value found by Finn et al. (2001) from $H S T$ /WFPC2 F606W data within the uncertainties. 


\section{$P G 1116+215, z=0.177$}

PSF problems affected the deep $\mathrm{H}$ band imaging of the host galaxy of this radio quiet object, with some conflicting results. Guvon et al. (2006) find that the host is disk-like and asymmetric with some evidence for a disturbance, while Veilleux et al. (2009) find that it shows no sprial arms or bar or morphological disturbance and classify it as an old merger, in agreement with the optical imaging (Bahcall et al. 1997).

There are $\sim 68$ galaxy clusters, groups, or candidates within this field around PG1116+215. The two with the smallest separations from the quasar $\left(0.28^{\prime}\right.$ and $\left.1.92^{\prime}\right)$ are $\mathrm{ZwCl} \mathrm{1116.5+2136} \mathrm{and} \mathrm{SDSSCGB} 16808$ (McConnachie et al. 2009), both with unknown redshifts. However, one cluster found in the SDSS data, MaxBCG J169.85817+21.20845, lies at $7.9^{\prime}$ separation and has a redshift marginally consistent with PG1116 $+215, z_{\text {phot }}=0.170$. The relatively large projected and redshift separations, $\sim 1 h^{-1} \mathrm{Mpc}$, and $\sim 1630 \mathrm{~km} \mathrm{~s}^{-1}$, place it toward the periphery of this cluster if it is associated with it at all.

Our results for $\mathrm{B}_{g q}$ from the 90Prime $r$ band image, 186 134 , is consistent with McLure \& Dunlop (2001b) and Finn et al. (2001) within the large statistical uncertainties. The $\mathrm{B}_{g q}$ values found in the $u$ and $g$ filters are also consistent with $\mathrm{B}_{g g}$, strengthening the conclusion that PG1116+215 lies in the outer regions or even outside the cluster potential of MaxBCG J169.85817+21.20845. However, the result for the $i$ band, $863 \pm 461$, presents a contradiction to this picture as it is greater than $\mathrm{B}_{g g}$, more consistent with an Abell class 1 environment (McLure \& Dunlop 2001b).

$$
P G 1307+085, z=0.155
$$

The host galaxy of this radio quiet quasar is an elliptical with no evidence of a disturbance (Bahcall et al. 1997; Hamilton et al. 2002; Guvon et al. 2006; Veilleux et al. 2009). The center of the $z=0.14$ cluster GMBCG J197.43444+08.33445 (Koester et al. 2007) lies at a projected separation of only $43.5^{\prime \prime}$ from the quasar. Given the redshift separation it is unlikely that PG1307+085 is a cluster member but many galaxies in the 90Prime field likely are.

Our results for $\mathrm{B}_{g q}$ in the $r$ band are consistent with McLure \& Dunlop (2001b) and Finn et al. (2001) within the large uncertainties. The $i$ band result for $\mathrm{B}_{g q}$ for this field is very large, $1461 \pm 778$. The $\mathrm{B}_{g q}$ value found for the $g$ band, $259 \pm 120$, is also large relative to other filters. The presence of GMBCG J197.43444+08.33445 in the foreground may be influencing the galaxy counts for this field, though it is not clear why this should only be the case for the $g$ and $i$ filters and not $u$ and $r$, which both show $\mathrm{B}_{g q}$ consistent with $\mathrm{B}_{g g}$.

$$
P G 1404+226, z=0.098, N L S 1
$$

$\mathrm{Ho}(2005)$ conclude that the host galaxy of this quasar has a very low star formation rate, $<0.14 \mathrm{M}_{\odot} \mathrm{yr}^{-1}$, based on the [O II] measurement of Kuraszkiewicz et al. (2000). It also has a low black hole mass, $\log \left(\mathrm{M}_{\mathrm{BH}} / \mathrm{M}_{\odot}\right)=6.88$, as estimated from the $\mathrm{H} \beta$ line width (Vestergaard \& Peterson 2006). McLeod \& Rieke (1994a) find that an exponential profile with a scale length of $2.98 \mathrm{~h}^{-1} \mathrm{kpc}$ gives a reasonable fit to their $\mathrm{H}$ band image of this AGN.

There are $\sim 50$ clusters, groups, or candidates identified in the $50^{\prime}$ around PG1404+226, including five compact groups of McConnachie et al. (2009) with separations less than $\sim 22^{\prime}$ and unknown redshift. The cluster WHL $\mathrm{J} 140609.9+221605$ has a separation of $8.17^{\prime}$ from the quasar and a redshift of $z=0.393$.

All values of $\mathrm{B}_{g q}$ for this field are consistent with $\mathrm{B}_{g g}$, except in the $g$ band, where we find $\mathrm{B}_{g q}=942 \pm 522$.

$$
P G 1444+407, z=0.267
$$

The morphology of the host galaxy of this radio quiet AGN is somewhat ambiguous. Bahcall et al. (1997) confirm the possible presence of a bar suggested by Hutchings \& Neff (1992) as the result of an old merger stage. Interestingly, based on the [O II] measurement of Baldwin et al. (1989), Ho (2005) infer a fairly high star formation rate in the host of $19.4 \mathrm{M}_{\odot} \mathrm{yr}^{-1}$, on the order of that found for interacting and luminous IR galaxies.

There are 48 galaxy clusters, groups, or candidates within $50^{\prime}$ of the quasar, with the closest, $\mathrm{ZwCl} 1443.8+4043$, lying at a separation of $12.6^{\prime}$, but unknown redshift.

For this field we find $\mathrm{B}_{g q}$ values in the $g, r$, and $i$ filters consistent with $\mathrm{B}_{g g}$. The $r$ band result is consistent with small positive values with large statistical uncertainties from the other studies of McLure \& Dunlop (2001b) and Finn et al. (2001). In the $u$ band, we find a $\mathrm{B}_{g q}<\mathrm{B}_{g g}$ by more than $2 \sigma$, reflecting the general trend for the $u$ band to show the smallest values of $\mathrm{B}_{g q}$, though we note that the limiting $u$ magnitude for this field corresponds to $\mathrm{M}^{*}+1.4$ at the redshift of this AGN rather than to $\mathrm{M}^{*}+2$.

$$
\text { PG1545+210 (3C 323.1), } z=0.264
$$

This FRII radio loud AGN has an elliptical host galaxy (McLeod \& Rieke 1994b; Bahcall et al. 1997) with a faint elliptical companion galaxy at a projected separation of $7.7 \mathrm{~h}^{-1} \mathrm{kpc}$. Ho (2005) finds a moderately large star formation rate of $9.7 \mathrm{M}_{\odot} \mathrm{yr}^{-1}$ in the host galaxy, based on the [O II] measurement of Wills et al. (1993). From population synthesis model fits to the optical spectrum of the companion, Canalizo \& Stockton (1997) report evidence of a possible interaction 2.3 Gyr ago. This quasar was found to have a rich cluster environment (Abell class 1) by Oemler et al. (1972), and Kirhakos et al. (1994) find 22 likely galaxies in their optical images described above in Sec. A.1. It is located $1.96^{\prime}$ away from another broad line AGN at a very similar redshift (SDSS J154749.70+205056.5, $z=0.26547$ ).

These AGNs are likely members of the X-ray indentified cluster ZwCl 1545.1+2104 $(z=0.266$, Piffaretti et al. (2011)), also found in the SDSS DR7 data $(z=0.26735$, Hao et al. (2010) $)$. There are $\sim 70$ other galaxy clusters, 
groups, or candidates within $50^{\prime}$ of PG1545+210. We note that this field also contains the highest number of $z<0.5$ AGN of any of the other 90Prime fields, listed in Table 9.

Here we a $\mathrm{B}_{g q}$ value in $g$ consistent with $\mathrm{B}_{g g}$, and the $r$ band value identically zero, as for MRK 586 . This is in agreement with the $H S T$ /WFPC2 F606W results of McLure \& Dunlop (2001b). However, given the smaller uncertainty quoted by Finn et al. (2001), our value $r$ band value differs from their positive results by $\sim 3 \sigma$. As for PG1307+085, we find a very large value of $\mathrm{B}_{g q}$ for this field in the $i$ band and a much smaller but significant signal in $u$, consistent with its known cluster environment. The limiting $u$ magnitude for this field corresponds to $\mathrm{M}^{*}+1.7$ at the redshift of this $\mathrm{AGN}$, rather than reaching to $\mathrm{M}^{*}+2$.

$$
P G 1612+261, z=0.131
$$

McLeod \& Rieke (1994a) find that an exponential profile with a scale length of $1.16 h^{-1} \mathrm{kpc}$ gives a reasonable fit to their H band image of this AGN and its immediate environment meets the criteria of McLeod \& Rieke (1994b) for bright interacting companion. (See Sec. A.3.)

It may be associated with the cluster NSC J161419+260832 $(z=0.1396)$ but given the velocity separation and the $\sim 0.5 h^{-1} \mathrm{Mpc}$ separation on the sky from the reported cluster center, it is likely on the periphery. There are $\sim 47$ other unique galaxy clusters, groups or candidates within $50^{\prime}$ of the quasar found in NED.

The $g$ band $\mathrm{B}_{g q}$ value for this field, $354 \pm 237$, is marginally greater than $\mathrm{B}_{g g}$, but it is consistent with $\mathrm{B}_{g g}$ in $u, r$ and $i$. The most closely separated $z<1$ QSO in this field, SDSS J161335.33+263127.7, lies at $z=0.708$ and $\sim 28.5^{\prime}$.

$$
\text { [HB89] 2141+175, z=0.211 }
$$

This radio loud AGN has an elliptical host, with some suggestion of an interacting companion (Hutchings \& Neff 1992; Dunlop et al. 2003). There is only one known galaxy cluster in this field, within $46^{\prime}$ of this quasar.

We have no $i$ band image from 90Prime to compare with the HST/WFPC2 F675W result of McLure \& Dunlop (2001b), but our large $r$ band result, $991 \pm 355$ is larger than theirs by $>2.5 \sigma$.

\section{REFERENCES}

Adelberger, K. L., \& Steidel, C. C. 2005, ApJ, 627, L1

Alonso, M. S., Lambas, D. G., Tissera, P., \& Coldwell, G. 2007, MNRAS, 375,1017

Bahcall, J. N., Kirhakos, S., Saxe, D. H., \& Schneider, D. P. 1997, ApJ, 479, 642

Baldwin, J. A., Wampler, E. J., \& Gaskell, C. M. 1989, ApJ, 338, 630

Barnes, J. E., \& Hernquist, L. 1992, ARA\&A, 30, 705

Barr, J. M., Bremer, M. N., Baker, J. C., \& Lehnert, M. D. 2003, MNRAS, 346, 229

Bennert, N., Canalizo, G., Jungwiert, B., et al. 2008, ApJ, 677, 846

Bertin, E., \& Arnouts, S. 1996, A\&AS, 117, 393

Bertin, E., Mellier, Y., Radovich, M., et al. 2002, in Astronomical Society of the Pacific Conference Series, Vol. 281, Astronomical Data Analysis Software and Systems XI, ed. D. A. Bohlender, D. Durand, \& T. H. Handley, 228

Best, P. N., Kauffmann, G., Heckman, T. M., et al. 2005 MNRAS, 362, 25

Blanton, M. R., \& Roweis, S. 2007, AJ, 133, 734

Brown, M. J. I., Boyle, B. J., \& Webster, R. L. 2001, AJ, 122, 26

Canalizo, G., Bennert, N., Jungwiert, B., et al. 2007, ApJ, 669, 801

Canalizo, G., \& Stockton, A. 1997, ApJ, 480, L5

Coil, A. L., Hennawi, J. F., Newman, J. A., Cooper, M. C., \& Davis, M. 2007, ApJ, 654, 115

Coil, A. L., Newman, J. A., Kaiser, N., et al. 2004, ApJ, 617, 765

Coil, A. L., Georgakakis, A., Newman, J. A., et al. 2009, ApJ, 701,1484

Coldwell, G. V., Gurovich, S., Díaz Tello, J., Söchting, I. K., \& Lambas, D. G. 2014, MNRAS, 437, 1199

Coldwell, G. V., \& Lambas, D. G. 2003, MNRAS, 344, 156

-. 2006, MNRAS, 371, 786

Coldwell, G. V., Martínez, H. J., \& Lambas, D. G. 2002,

MNRAS, 336, 207

Constantin, A., Hoyle, F., \& Vogeley, M. S. 2008, ApJ, 673, 715

Constantin, A., \& Vogeley, M. S. 2006, ApJ, 650, 727

Cool, R. J., Eisenstein, D. J., Kochanek, C. S., et al. 2012, ApJ, 748,10

Croom, S. M., \& Shanks, T. 1999, MNRAS, 303, 411

Croom, S. M., Boyle, B. J., Shanks, T., et al. 2005, MNRAS, 356 415

Croton, D. J., Springel, V., White, S. D. M., et al. 2006, MNRAS, 365,11

Davis, M., \& Peebles, P. J. E. 1983, ApJ, 267, 465

Di Matteo, T., Springel, V., \& Hernquist, L. 2005, Nature, 433, 604

Dressler, A. 1980, ApJ, 236, 351

Dunlop, J. S., McLure, R. J., Kukula, M. J., et al. 2003, MNRAS, 340,1095
Ellingson, E., Yee, H. K. C., \& Green, R. F. 1991, ApJ, 371, 49

Ellison, S. L., Mendel, J. T., Patton, D. R., \& Scudder, J. M. 2013, MNRAS, 435, 3627

Faber, S. M., Willmer, C. N. A., Wolf, C., et al. 2007, ApJ, 665, 265

Ferrarese, L., \& Merritt, D. 2000, ApJ, 539, L9

Finn, R. A., Impey, C. D., \& Hooper, E. J. 2001, ApJ, 557, 578

Fisher, K. B., Bahcall, J. N., Kirhakos, S., \& Schneider, D. P. 1996, ApJ, 468, 469

Floyd, D. J. E., Kukula, M. J., Dunlop, J. S., et al. 2004, MNRAS, 355, 196

Gabor, J. M., Impey, C. D., Jahnke, K., et al. 2009, ApJ, 691, 705

Gebhardt, K., Bender, R., Bower, G., et al. 2000, ApJ, 539, L13

Gilli, R., Cimatti, A., Daddi, E., et al. 2003, ApJ, 592, 721

Gilli, R., Zamorani, G., Miyaji, T., et al. 2009, A\&A, 494, 33

Granato, G. L., De Zotti, G., Silva, L., Bressan, A., \& Danese, L. 2004, ApJ, 600, 580

Grogin, N. A., Conselice, C. J., Chatzichristou, E., et al. 2005, ApJ, 627, L97

Groth, E. J., \& Peebles, P. J. E. 1977, ApJ, 217, 385

Gunn, J. E., \& Stryker, L. L. 1983, ApJS, 52, 121

Guyon, O., Sanders, D. B., \& Stockton, A. 2006, ApJS, 166, 89

Haiman, Z., Ciotti, L., \& Ostriker, J. P. 2004, ApJ, 606, 763

Hall, P. B., Green, R. F., \& Cohen, M. 1998, ApJS, 119, 1

Hamilton, T. S., Casertano, S., \& Turnshek, D. A. 2002, ApJ, 576,61

Hao, J., McKay, T. A., Koester, B. P., et al. 2010, ApJS, 191, 254

Heckman, T. M., Bothun, G. D., Balick, B., \& Smith, E. P. 1984, AJ, 89,958

Hennawi, J. F., Strauss, M. A., Oguri, M., et al. 2006, AJ, 131, 1

Hickox, R. C., Jones, C., Forman, W. R., et al. 2009, ApJ, 696, 891

Ho, L. C. 2005, ApJ, 629, 680

Ho, L. C., \& Kim, M. 2009, ApJS, 184, 398

Hopkins, P. F., \& Hernquist, L. 2006, ApJS, 166, 1

Hopkins, P. F., Hernquist, L., Cox, T. J., et al. 2006a, ApJS, 163, 1

Hopkins, P. F., Hernquist, L., Cox, T. J., \& Kereš, D. 2008, ApJS, 175,356

Hopkins, P. F., Somerville, R. S., Hernquist, L., et al. 2006b, ApJ, 652, 864

Hutchings, J. B., \& Neff, S. G. 1992, AJ, 104, 1

Infante, L. 1994, A\&A, 282, 353

Kauffmann, G., \& Haehnelt, M. 2000, MNRAS, 311, 576

Kauffmann, G., White, S. D. M., Heckman, T. M., et al. 2004 MNRAS, 353, 713

Kauffmann, G., Heckman, T. M., Tremonti, C., et al. 2003

MNRAS, 346, 1055

Kewley, L. J., Groves, B., Kauffmann, G., \& Heckman, T. 2006, MNRAS, 372, 961 
Kinney, A. L., Calzetti, D., Bohlin, R. C., et al. 1996, ApJ, 467, 38

Kirhakos, S., Sargent, W. L. W., Schneider, D. P., et al. 1994, PASP, 106,646

Koester, B. P., McKay, T. A., Annis, J., et al. 2007, ApJ, 660, 239

Kuraszkiewicz, J., Wilkes, B. J., Brandt, W. N., \& Vestergaard, M. 2000, ApJ, 542, 631

Labita, M., Treves, A., Falomo, R., \& Uslenghi, M. 2006, MNRAS, 373, 551

Landy, S. D., \& Szalay, A. S. 1993, ApJ, 412, 64

Li, C., Kauffmann, G., Heckman, T. M., White, S. D. M., \& Jing, Y. P. 2008, MNRAS, 385, 1915

Li, C., Kauffmann, G., Wang, L., et al. 2006, MNRAS, 373, 457

Limber, D. N. 1953, ApJ, 117, 134

Longair, M. S., \& Seldner, M. 1979, MNRAS, 189, 433

Loveday, J., Norberg, P., Baldry, I. K., et al. 2012, MNRAS, 420 , 1239

Lupton, R. H., Gunn, J. E., \& Szalay, A. S. 1999, AJ, 118, 1406

Marconi, A., \& Hunt, L. K. 2003, ApJ, 589, L21

McConnachie, A. W., Patton, D. R., Ellison, S. L., \& Simard, L. 2009, MNRAS, 395, 255

McLeod, K. K., \& Rieke, G. H. 1994a, ApJ, 420, 58

-. 1994b, ApJ, 431, 137

- 1995, ApJ, 454, L77

McLeod, K. K., Rieke, G. H., \& Storrie-Lombardi, L. J. 1999, ApJ, 511, L67

McLure, R. J., \& Dunlop, J. S. 2001a, MNRAS, 327, 199

—. 2001b, MNRAS, 321, 515

- 2002, MNRAS, 331,795

McLure, R. J., Kukula, M. J., Dunlop, J. S., et al. 1999, MNRAS, 308,377

Montero-Dorta, A. D., Croton, D. J., Yan, R., et al. 2009, MNRAS, 392, 125

Myers, A. D., Brunner, R. J., Nichol, R. C., et al. 2007a, ApJ, 658,85

Myers, A. D., Brunner, R. J., Richards, G. T., et al. 2007b, ApJ, 658,99

-. 2006, ApJ, 638, 622

O'Donnell, J. E. 1994, ApJ, 422, 158

Oemler, Jr., A., Gunn, J. E., \& Oke, J. B. 1972, ApJ, 176, L47

Peterson, B. M., Ferrarese, L., Gilbert, K. M., et al. 2004, ApJ, 613,682

Piffaretti, R., Arnaud, M., Pratt, G. W., Pointecouteau, E., \& Melin, J.-B. 2011, A\&A, 534, A109

Porciani, C., Magliocchetti, M., \& Norberg, P. 2004, MNRAS, 355,1010

Porciani, C., \& Norberg, P. 2006, MNRAS, 371, 1824

Prescott, M., Baldry, I. K., \& James, P. A. 2009, MNRAS, 397, 90

Ramos Almeida, C., Bessiere, P. S., Tadhunter, C., et al. 2013, ArXiv e-prints, arXiv:1308.4725

Ross, N. P., Shen, Y., Strauss, M. A., et al. 2009, ApJ, 697, 1634

Sabater, J., Best, P. N., \& Argudo-Fernández, M. 2013, MNRAS, 430,638
Sabater, J., Verdes-Montenegro, L., Leon, S., Best, P., \& Sulentic, J. 2012, A\&A, 545, A15

Sanders, D. B., Soifer, B. T., Elias, J. H., et al. 1988, ApJ, 325, 74

Schlafly, E. F., \& Finkbeiner, D. P. 2011, ApJ, 737, 103

Serber, W., Bahcall, N., Ménard, B., \& Richards, G. 2006, ApJ, 643,68

Shang, Z., Wills, B. J., Wills, D., \& Brotherton, M. S. 2007, AJ, 134,294

Shen, Y., Strauss, M. A., Oguri, M., et al. 2007, AJ, 133, 2222

Shen, Y., Strauss, M. A., Ross, N. P., et al. 2009, ApJ, 697, 1656

Shen, Y., Hennawi, J. F., Shankar, F., et al. 2010, ApJ, 719, 1693

Silverman, J. D., Mainieri, V., Lehmer, B. D., et al. 2008, ApJ, 675,1025

Silverman, J. D., Kovač, K., Knobel, C., et al. 2009, ApJ, 695, 171

Smith, R. J., Boyle, B. J., \& Maddox, S. J. 1995, MNRAS, 277, 270

Söchting, I. K., Clowes, R. G., \& Campusano, L. E. 2002, MNRAS, 331, 569

-. 2004, MNRAS, 347, 1241

Somerville, R. S., Hopkins, P. F., Cox, T. J., Robertson, B. E., \&

Hernquist, L. 2008, MNRAS, 391, 481

Springel, V., Di Matteo, T., \& Hernquist, L. 2005a, ApJ, 620, L79

. 2005b, MNRAS, 361, 776

Stockton, A., \& MacKenty, J. W. 1987, ApJ, 316, 584

Teng, S. H., \& Veilleux, S. 2010, ApJ, 725, 1848

Teplitz, H. I., McLean, I. S., \& Malkan, M. A. 1999, ApJ, 520, 469

Tremaine, S., Gebhardt, K., Bender, R., et al. 2002, ApJ, 574, 740

Vanden Berk, D. E., Richards, G. T., Bauer, A., et al. 2001, AJ, 122,549

Veilleux, S., Rupke, D. S. N., Kim, D.-C., et al. 2009, ApJS, 182, 628

Véron-Cetty, M.-P., \& Véron, P. 2010, A\&A, 518, A10

Vestergaard, M., \& Peterson, B. M. 2006, ApJ, 641, 689

Volonteri, M., Haardt, F., \& Madau, P. 2003, ApJ, 582, 559

Wake, D. A., Miller, C. J., Di Matteo, T., et al. 2004, ApJ, 610, L85

Wilkes, B. J., Kuraszkiewicz, J., Green, P. J., Mathur, S., \& McDowell, J. C. 1999, ApJ, 513, 76

Williams, G. G., Olszewski, E., Lesser, M. P., \& Burge, J. H. 2004 , in Society of Photo-Optical Instrumentation Engineers (SPIE) Conference Series, Vol. 5492, Ground-based Instrumentation for Astronomy, ed. A. F. M. Moorwood \& M. Iye, $787-798$

Wills, B. J., Netzer, H., Brotherton, M. S., et al. 1993, ApJ, 410, 534

Wold, M., Armus, L., Neugebauer, G., Jarrett, T. H., \& Lehnert, M. D. 2003, AJ, 126, 1776

Wold, M., Lacy, M., Lilje, P. B., \& Serjeant, S. 2001, MNRAS, 323,231

Wyithe, J. S. B., \& Loeb, A. 2003, ApJ, 595, 614

Yates, M. G., Miller, L., \& Peacock, J. A. 1989, MNRAS, 240, 129

Yee, H. K. C., \& Ellingson, E. 1993, ApJ, 411, 43

Yee, H. K. C., \& Green, R. F. 1984, ApJ, 280, 79

Yee, H. K. C., \& López-Cruz, O. 1999, AJ, 117, 1985 